Western University

Scholarship@Western

Chemistry Publications

Chemistry Department

Fall 9-29-2017

\title{
Aluminum Complexes of N2O23- Formazanate Ligands Supported by Phosphine Oxide Donors
}

Ryan Maar

Amir Rabiee Kenaree

Ruizhong Zhang

Yichen Tao

Benjamin D. Katzman

See next page for additional authors

Follow this and additional works at: https://ir.lib.uwo.ca/chempub

Part of the Chemistry Commons

Citation of this paper:

Maar, Ryan; Rabiee Kenaree, Amir; Zhang, Ruizhong; Tao, Yichen; Katzman, Benjamin D.; Staroverov, Viktor N.; Ding, Zhifeng; and Gilroy, Joe, "Aluminum Complexes of N2O23- Formazanate Ligands Supported by Phosphine Oxide Donors" (2017). Chemistry Publications. 81.

https://ir.lib.uwo.ca/chempub/81 
Authors

Ryan Maar, Amir Rabiee Kenaree, Ruizhong Zhang, Yichen Tao, Benjamin D. Katzman, Viktor N. Staroverov, Zhifeng Ding, and Joe Gilroy 


\section{Aluminum Complexes of $\mathrm{N}_{2} \mathrm{O}_{2}{ }^{3-}$ Formazanate Ligands Supported by Phosphine Oxide Donors}

Ryan R. Maar, Amir Rabiee Kenaree, Ruizhong Zhang, Yichen Tao, Benjamin D. Katzman, Viktor N. Staroverov, Zhifeng Ding, Joe B. Gilroy*

Department of Chemistry and The Centre for Advanced Materials and Biomaterials Research (CAMBR), The University of Western Ontario, 1151 Richmond St. N., London, Ontario, Canada, N6A 5B7. Telephone: +1-519-661-2111 ext. 81561 (JBG). E-mail: joe.gilroy@uwo.ca 


\section{ABSTRACT}

The synthesis and characterization of a new family of phosphine-oxide-supported aluminum formazanate complexes $(\mathbf{7 a}, \mathbf{7 b}, \mathbf{8 a}, \mathbf{9 a})$ are reported. X-ray diffraction studies revealed that the aluminum atoms in the complexes adopt an octahedral geometry in the solid state. The equatorial positions are occupied by an $\mathrm{N}_{2} \mathrm{O}_{2}{ }^{3-}$ formazanate ligand, and the axial positions are occupied by L-type phosphine oxide donors. UV-vis absorption spectroscopy revealed that the complexes were strongly absorbing $\left(\varepsilon \sim 30,000 \mathrm{M}^{-1} \mathrm{~cm}^{-1}\right)$ between 500 and $700 \mathrm{~nm}$. The absorption maxima in this region were simulated using time-dependent density-functional theory. With the exception of 3-cyano substituted complex $\mathbf{7 b}$, which showed maximum luminescence intensity in the presence of excess phosphine oxide, the title complexes are non-emissive in solution and the solid state. The electrochemical properties of the complexes were probed using cyclic voltammetry. Each complex underwent sequential one-electron oxidations in potential ranges of -0.12 to $0.29 \mathrm{~V}$ and 0.62 to $0.97 \mathrm{~V}$, relative to the ferrocene/ferrocenium redox couple. Electrochemical reduction events were observed at potentials between -1.34 and $-1.75 \mathrm{~V}$. When combined with tri-n-propylamine as a coreactant, complex $\mathbf{7 b}$ acted as an electrochemiluminescence emitter with a maximum electrochemiluminescence intensity at a wavelength of $735 \mathrm{~nm}$, red-shifted relative to the photoluminescence maximum of the same compound.

\section{INTRODUCTION}

Aluminum coordination chemistry is an attractive route to functional molecular materials for researchers working in numerous fields, including catalysis ${ }^{1-5}$ and organic photovoltaics, ${ }^{6-9}$ due to the low cost of aluminum $\left(\sim 2.00 \mathrm{~kg}^{-1}\right)$ and its high natural abundance $(\sim 8 \%$ of Earth's crust). ${ }^{10}$ Generally, multidentate chelating ligands such as functionalized $\alpha$-diiminates, ${ }^{11-12} 8$ hydroxylquinolinates, ${ }^{7-9}$ salen, ${ }^{13-14}$ bis(iminopyridines),${ }^{15-16}$ and dipyrrinates ${ }^{17-18}$ are used as 
scaffolds to generate aluminum(III) coordination complexes, with many examples possessing unique electrochemical and spectroscopic properties. For instance, Berben and co-workers synthesized bis(iminopyridine) complex 1, which generates $\mathrm{H}_{2}$ from $\mathrm{H}^{+}$upon application of a reducing potential. ${ }^{19}$ The Coates group produced fluorinated complex $\mathbf{2}$, which proved to be an effective catalyst for the polymerization of tricyclic anhydrides with minimal side reactivity. ${ }^{20}$ The Nabeshima group synthesized dipyrrinate complex 3, which exhibited colourimetric and fluorometric responses to alkaline earth metals. ${ }^{21} \mathrm{Ma}$ and colleagues designed $\beta$-diketiminate complex $\mathbf{4}$ and demonstrated its use as an effective catalyst for the ring-opening polymerization of cyclic esters. ${ }^{22}$ The aforementioned complexes utilize a $\pi$-conjugated, multidentate ligand with at least two nitrogen atoms chelated to aluminum. Another class of $N$-donor ligands that are structurally related to $\beta$-diketiminates ${ }^{23}(\mathbf{5})$ are formazanates $(\mathbf{6}){ }^{24}$

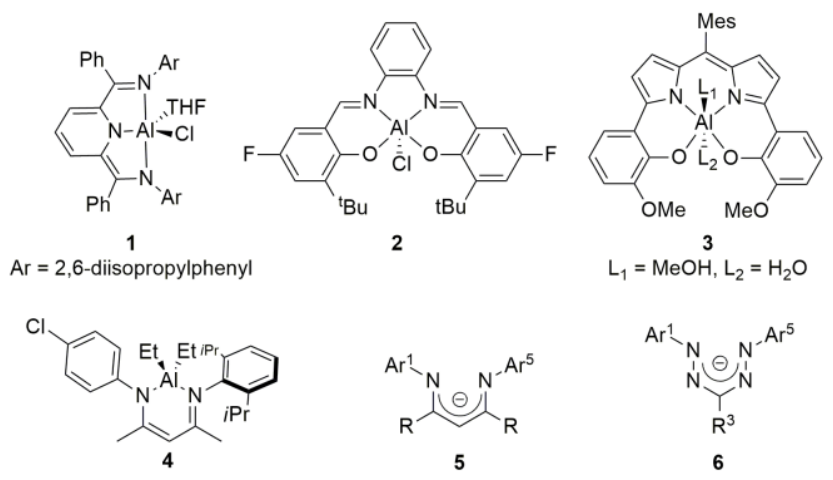

Transition metal complexes of formazanates have attracted considerable interest due to the redox-active nature of the formazanate scaffold, ${ }^{25-27}$ their spectroscopic activity within the visible region of the electromagnetic spectrum, ${ }^{28}$ and unique magnetic properties. ${ }^{29-30}$ Boron difluoride $\left(\mathrm{BF}_{2}\right)$ complexes of formazanate ligands have been extensively studied by the Gilroy $^{31}$ and Otten ${ }^{32}$ groups. These compounds have been used as fluorescent cell-imaging agents, ${ }^{33}$ acted as precursors to unusual boron-based heterocycles, ${ }^{34-35}$ and employed as 
electrochemiluminescence (ECL) emitters. ${ }^{36}$ By contrast, the coordination chemistry of formazanate ligands and heavier group-13 elements (i.e., $\mathrm{Al}, \mathrm{Ga}, \mathrm{In}$ ) has been investigated to a lesser extent. ${ }^{37}$ In an effort to further develop group-13 formazanate chemistry, we report the synthesis and physical characterization of a series of hexacoordinate aluminum complexes bearing two L-type phosphine oxide donors and a tetradentate $\mathrm{N}_{2} \mathrm{O}_{2}{ }^{3-}$ formazanate ligands. In doing so, we demonstrate the potential of this class of functional materials for use in optoelectronic applications.

\section{EXPERIMENTAL SECTION}

\section{General Considerations}

Reactions and manipulations were carried out under an $\mathrm{N}_{2}$ atmosphere using standard glove box or Schlenk techniques unless otherwise stated. Reagents were purchased from SigmaAldrich or Alfa Aesar, stored at room temperature, and used as received unless otherwise specified. Tri- $n$-propylamine (TPrA) was stored at $4{ }^{\circ} \mathrm{C}$. Solvents were purchased from Caledon Laboratories, dried using an Innovative Technologies Inc. solvent purification system, collected under vacuum, and stored under an $\mathrm{N}_{2}$ atmosphere over $4 \AA$ molecular sieves. Tri(ethylferrocene)phosphine ${ }^{38}$ and formazan $\mathbf{6}^{39}$ were synthesized according to literature procedures. NMR spectra were recorded on $400 \mathrm{MHz}\left({ }^{1} \mathrm{H}: 399.8 \mathrm{MHz} ;{ }^{13} \mathrm{C}\left\{{ }^{1} \mathrm{H}\right\}: 100.5 \mathrm{MHz}\right)$ or $600 \mathrm{MHz}\left({ }^{1} \mathrm{H}: 599.3 \mathrm{MHz} ;{ }^{13} \mathrm{C}\left\{{ }^{1} \mathrm{H}\right\}: 150.7 \mathrm{MHz},{ }^{31} \mathrm{P}: 242.6 \mathrm{MHz}\right)$ Varian INOVA spectrometers at $25{ }^{\circ} \mathrm{C}$ or at variable temperatures. ${ }^{1} \mathrm{H}$ NMR spectra were referenced to residual $\mathrm{CHCl}_{3}(\delta=$ 7.27) or $\mathrm{C}_{6} \mathrm{D}_{5} \mathrm{H}(\delta=7.16)$, and ${ }^{13} \mathrm{C}\left\{{ }^{1} \mathrm{H}\right\}$ NMR spectra were referenced to $\mathrm{CDCl}_{3}(\delta=77.0)$ or $\mathrm{C}_{6} \mathrm{D}_{6}(\delta=128.1) .{ }^{31} \mathrm{P}$ NMR spectra were referenced with respect to $\mathrm{H}_{3} \mathrm{PO}_{4}(\delta=0)$. Massspectrometry data were recorded in positive-ion mode using a high-resolution Finnigan MAT 8200 spectrometer using electron impact ionization or a Micromass LCT electrospray time-of- 
flight mass-spectrometer. UV-vis absorption spectra were recorded using a Cary 5000 UV-VisnIR spectrophotometer for solutions of the analyte in the absence/presence of excess phosphine oxide. Molar extinction coefficients were determined from the slope of a plot of absorbance against concentration using four solutions with known concentrations ranging between 5 and 50 $\mu \mathrm{M}$. Photoluminescence (PL) spectra of $0.08 \mathrm{mM} \mathrm{CH}_{2} \mathrm{Cl}_{2}$ solutions were recorded on a Photon Technology International (QM-7/2005) spectrometer at an excitation wavelength of $467 \mathrm{~nm}$. Fluorescence quantum yields $\left(\Phi_{\mathrm{F}}, \%\right)$ were determined using equation 1:

$$
\Phi_{\mathrm{F}}=\frac{I_{\mathrm{x}}}{I_{\mathrm{st}}} \frac{A_{\mathrm{st}}}{A_{\mathrm{x}}}\left(\frac{\eta_{\mathrm{x}}}{\eta_{\mathrm{st}}}\right)^{2} \times 100 \%
$$

where $I$ is the integrated fluorescence intensity, $A$ is the optical density and $\eta$ is the refractive index of the solvent. The subscript "st" refers to standard and " $\mathrm{x}$ " refers to the analyte. Infrared spectra were recorded on a PerkinElmer Spectrum Two instrument using an attenuated total reflectance accessory. The relative standard employed was $\left[\mathrm{Ru}(\mathrm{bpy})_{3}\right]\left[\mathrm{PF}_{6}\right]_{2}$ in $\mathrm{CH}_{3} \mathrm{CN}\left(\Phi_{\mathrm{F}}=\right.$ $9.5 \%) .{ }^{40}$ Elemental analyses $(\mathrm{C}, \mathrm{H}, \mathrm{N})$ were carried out by Laboratoire d'Analyse Élémentaire de l’Université de Montréal, Montréal, QC, Canada and Canadian Microanalytical Services Ltd., Delta, BC, Canada.

\section{Purity of new compounds}

The purity of new compounds described in this study was demonstrated by providing clean ${ }^{1} \mathrm{H}$, ${ }^{13} \mathrm{C}\left\{{ }^{1} \mathrm{H}\right\}$, and ${ }^{31} \mathrm{P}$ NMR spectra, high-resolution mass-spectrometry, and elemental analysis data. For complexes $\mathbf{7 a}, \mathbf{7 b}$, and $\mathbf{8 a}$, elemental analysis for carbon was consistently low, while $\mathrm{H}$ and $\mathrm{N}$ analysis matched well across several independent batches. However, these data are provided to illustrate the best values obtained to date. 


\section{Cyclic Voltammetry}

Cyclic voltammetry experiments were performed in an Ar-filled glovebox using a Bioanalytical Systems Inc. (BASi) Epsilon potentiostat and analyzed using BASi Epsilon software. Typical electrochemical cells consisted of a three-electrode setup including a silver pseudo-reference electrode, glassy carbon working electrode, and platinum counter-electrode. Experiments were run at a scan rate of $0.25 \mathrm{~V} \mathrm{~s}^{-1}$ in dry and degassed $\mathrm{CH}_{2} \mathrm{Cl}_{2}$ solutions of the analyte $(\sim 1 \mathrm{mM})$, phosphine oxide $(\sim 50 \mathrm{mM})$, and supporting electrolyte $\left(0.1 \mathrm{M}\left[n \mathrm{Bu}_{4} \mathrm{~N}\right]\left[\mathrm{PF}_{6}\right]\right)$. Cyclic voltammograms were referenced relative to the ferrocene/ferrocenium or decamethylferrocene/decamethylferrocenium redox couples $(\sim 1 \mathrm{mM}$ internal standard $)$ and corrected for internal cell resistance using the BASi Epsilon software. The decamethylferrocene/decamethylferrocenium oxidation wave was found at $-0.55 \mathrm{~V}$ relative to ferrocene/ferrocenium under identical conditions.

\section{Electrochemiluminescence Measurements}

Electrochemiluminescence (ECL) experiments were carried out in a conventional three-electrode cell at room temperature in a $25 \mathrm{~cm}$ length glass cylinder cell with an inner diameter of $2.5 \mathrm{~cm}$ and equipped with a flat Pyrex window at the bottom for light detection. A $2 \mathrm{~mm}$ diameter platinum disk inlaid in a glass sheath and two coiled platinum wires were used as working, counter-, and pseudo-reference electrodes. Before each experiment, the working electrode was polished carefully with 0.1 and $0.05 \mu \mathrm{m}$ alumina slurry on a cloth pad to obtain a mirror finish and washed repeatedly with deionized water. The working electrode was then electrochemically polished in $0.1 \mathrm{M} \mathrm{H}_{2} \mathrm{SO}_{4}$ between -1.0 and $1.0 \mathrm{~V}$ at a scan rate of $0.5 \mathrm{~V} \mathrm{~s}^{-1}$ to obtain a clean and reproducible Pt surface. The working electrode was finally washed repeatedly with deionized $\mathrm{H}_{2} \mathrm{O}$ and dried with a stream of Ar. The counter- and reference-electrodes were sonicated in 
deionized $\mathrm{H}_{2} \mathrm{O}$ for 5 min before they were thoroughly rinsed with deionized $\mathrm{H}_{2} \mathrm{O}$, dried at $120{ }^{\circ} \mathrm{C}$ for $12 \mathrm{~h}$, and cooled to room temperature before use. For coreactant ECL experiments, $5 \mathrm{mM}$ TPrA was added to a $0.6 \mathrm{mM}$ solution of $7 \mathbf{b}$ in dry $\mathrm{CH}_{2} \mathrm{Cl}_{2}$ containing $30 \mathrm{mM} \mathrm{OPPh}_{3}$ and $0.1 \mathrm{M}$ $\left[n \mathrm{Bu}_{4} \mathrm{~N}\right]\left[\mathrm{PF}_{6}\right]$ as the supporting electrolyte and degassed with Ar to remove dissolved oxygen. The electrochemical potential window was calibrated after the experiment using the ferrocene/ferrocenium redox couple as an internal standard.

ECL experiments were conducted using a $\mathrm{CH}$ Instruments (CHI 610A) electrochemical analyzer. The ECL intensity was detected as a photocurrent by a Hamamatsu photomultiplier tube (R928) and transformed to a voltage signal using a Keithley 6487 picoammeter/voltage source. The sensitivity setting on the picoammeter was set manually to avoid photosaturation. The potentials, current signals from the electrochemical analyzer, and the ECL photocurrent signal from the picoammeter were sent simultaneously through a National Instruments DAQ data acquisition board (DAQ 6052E) to a computer. The data acquisition system was controlled from a custom-made National Instruments LabVIEW program (ECL-PMT610a.vi). Accumulated and spooling ECL spectra were obtained by placing the ECL cell onto a Princeton Instruments Acton 2300i spectrometer with a grating of $50 \mathrm{lmm}^{-1}$ blazed at $600 \mathrm{~nm}$ and an Andor Technology CCD camera (Andor iDUS, model DU401-BR-DD-352) cooled at $-65^{\circ} \mathrm{C}$. The system was calibrated before each experiment using an Ocean Optics Mercury-Argon lamp. The exposure time and kinetic series lengths were optimized to produce the clearest ECL spectra. The CHI 610A electrochemical analyzer and the Andor technology programs were run simultaneously. Accumulated ECL spectra were recorded during four successive scan segments in each experiment. For the spooling ECL spectroscopy studies, the following parameters for a cyclic potential scan at $0.025 \mathrm{~V} \mathrm{~s}^{-1}$ in the range between -0.24 and $1.26 \mathrm{~V}$, for instance, were used for the Andor Technology program under the kinetic parameters option tab: exposure time $=2 \mathrm{~s}$, 
number of accumulations $=1$, kinetic series length $=60$, kinetic cycle time $=1$, and the spectrometer was centered at $546 \mathrm{~nm}$ using the $50 \mathrm{lmm}^{-1}$ grating. During ECL experiments, the lights in the experimentation room were switched off and blackout curtains were employed to reduce background interference.

ECL efficiency, $\Phi_{\mathrm{ECL}}(\%)$, was determined by comparing the ratio of the integrated ECL intensity to the charge of the analyte in reference to that of $\left[\mathrm{Ru}(\mathrm{bpy})_{3}\right]\left[\mathrm{PF}_{6}\right]_{2} / \mathrm{TPrA},{ }^{41-44} \mathrm{using}$ equation (2):

$$
\Phi_{\mathrm{ECL}}=\frac{\left(\frac{\int \mathrm{ECL} d t}{\int \text { Current } d t}\right)_{\mathrm{x}}}{\left(\frac{\int \mathrm{ECL} d t}{\int \text { Current } d t}\right)_{\mathrm{st}}} \times 100 \%
$$

where "ECL" and "Current" represent ECL intensity and electrochemical current values respectively, "st" refers to the $\left[\mathrm{Ru}(\mathrm{bpy})_{3}\right]\left[\mathrm{PF}_{6}\right]_{2} / \mathrm{TPrA}$ standard and "x" refers to the analyte.

\section{X-ray Crystallography Methods}

Single crystals for X-ray diffraction studies were grown by vapor diffusion of pentane into a saturated solution of the compound in $\mathrm{CH}_{2} \mathrm{Cl}_{2}(\mathbf{7 a}, 7 \mathbf{b}, \mathbf{8 a}$, and 9a). Samples were mounted on a MiTeGen polyimide micromount with a small amount of Paratone $N$ oil. X-ray diffraction measurements were made on a Bruker Kappa Axis Apex2 (7a and 8a) or Nonius KappaCCD Apex2 (7b and 9a) diffractometer at a temperature of $110 \mathrm{~K}$. The data collection strategy involved a number of $\omega$ and $\varphi$ scans which allowed for data acquisition over a range of angles, 20. The frame integration was performed using SAINT. ${ }^{45}$ The resulting raw data was scaled and absorption corrected using a multi-scan averaging of symmetry equivalent data using SADABS. $^{46}$ The structure was solved by using a dual space methodology using the SHELXT program. ${ }^{47}$ All non-hydrogen atoms were obtained from the initial solution. The hydrogen atoms were introduced at idealized positions and were allowed to ride on the parent atom. The 
structural model was fit to the data using full matrix least-squares based on $F^{2}$. The calculated structure factors included corrections for anomalous dispersion from the usual tabulation. The electron density difference map of $\mathbf{9 a}$ showed regions that could not be modeled accurately. Thus, the PLATON SQUEEZE program ${ }^{48}$ was used, and analysis was continued on these data. The structure was refined using the SHELXL-2014 program from the SHELXTL program package. $^{49}$ See Table S1 and CCDC 1548211-1548213 and 1553985 for additional crystallographic data.

\section{1,5-bis(2-hydroxyphenyl)-3-phenylformazan}

In air, phenylpyruvic acid $(1.07 \mathrm{~g}, 6.52 \mathrm{mmol})$ and sodium hydroxide $(2.68 \mathrm{~g}, 67.0 \mathrm{mmol})$ were dissolved in deionized water $(150 \mathrm{~mL})$. The pale-yellow solution was cooled to $-10{ }^{\circ} \mathrm{C}$ in an acetone/ice bath and stirred for $30 \mathrm{~min}$. In a separate flask, 2-aminophenol (2.02 g, $18.5 \mathrm{mmol})$ was combined with concentrated sulfuric acid $(5.52 \mathrm{~g}, 3.00 \mathrm{~mL}, 56.3 \mathrm{mmol})$ in deionized water $(13 \mathrm{~mL})$ and stirred for $15 \mathrm{~min}$ at $-10^{\circ} \mathrm{C}$. A solution of sodium nitrite $(1.34 \mathrm{~g}, 19.4 \mathrm{mmol})$ in deionized water $(6 \mathrm{~mL})$ was also cooled to $-10{ }^{\circ} \mathrm{C}$ for $15 \mathrm{~min}$ before it was added dropwise over a $15 \mathrm{~min}$ period to the 2 -aminophenol solution. The resulting red/brown diazonium salt solution was stirred for $10 \mathrm{~min}$ at $-10{ }^{\circ} \mathrm{C}$ before it was added dropwise to the alkaline phenylpyruvic acid solution described above over a 15 min period. Upon addition, the solution turned dark $\mathrm{red} /$ purple and a precipitate of the same colour formed. The mixture was stirred for $3 \mathrm{~h}$ before the precipitate was collected by vacuum filtration to afford a dark-purple solid. This solid was dissolved in EtOAc and transferred to a neutral alumina column $\left(1.0^{\prime \prime} \times 3.0^{\prime \prime}\right)$. Using EtOAc as eluent, 1,5-bis(2-hydroxyphenyl)-3-phenylformazan $\left(R_{f}=0.95\right)$ was isolated from the column and concentrated in vacuo. The dark-purple solid was triturated with chilled $\mathrm{MeOH}(\sim 15 \mathrm{~mL})$ and collected via vacuum filtration to afford 1,5-bis(2-hydroxyphenyl)-3-phenylformazan as a dark-purple microcrystalline solid. Yield $=0.21$ g, 10\%. M.p.: $166-168{ }^{\circ} \mathrm{C} .{ }^{1} \mathrm{H}$ NMR $(599.3$ 
$\left.\mathrm{MHz}, \mathrm{CDCl}_{3}\right): \delta 15.65(\mathrm{~s}, 1 \mathrm{H}, \mathrm{N} \underline{H}), 11.02(\mathrm{~s}, 2 \mathrm{H}, \mathrm{O} \underline{H}), 7.80\left(\mathrm{~d}, J_{\mathrm{HH}}=7 \mathrm{~Hz}, 2 \mathrm{H}, \operatorname{aryl} \mathrm{C} \underline{H}\right), 7.48$ $\left(\mathrm{t}, J_{\mathrm{HH}}=8 \mathrm{~Hz}, 2 \mathrm{H}, \operatorname{aryl} \mathrm{C} \underline{H}\right), 7.43-7.41(\mathrm{~m}, 3 \mathrm{H}, \operatorname{aryl} \mathrm{C} \underline{H}), 7.22-7.19(\mathrm{~m}, 2 \mathrm{H}, \operatorname{aryl~C} \underline{H}), 7.03-7.00$ (m, 4H, aryl $\mathrm{C} \underline{H}) .{ }^{13} \mathrm{C}\left\{{ }^{1} \mathrm{H}\right\}$ NMR $\left(150.7 \mathrm{MHz}, \mathrm{CDCl}_{3}\right): \delta 150.4,138.9,135.5,132.3,129.3$, 129.0, 128.6, 125.3, 124.1, 120.6, 118.3. FT-IR (ATR): 3314 (br, s), 3057 (w), 1601 (m), 1581 (m), 1589 (s), 1507 (s), 1463 (s), 1222 (s), 1208 (s), 1147 (s), 1031 (m) 737 (s), 686 (s), 649 (s), $605(\mathrm{~s}), 579(\mathrm{~m}), 536(\mathrm{~m}) \mathrm{cm}^{-1}$. UV-vis $\left(\mathrm{CH}_{2} \mathrm{Cl}_{2}\right): \lambda_{\max }=558 \mathrm{~nm}\left(\varepsilon=16,300 \mathrm{M}^{-1} \mathrm{~cm}^{-1}\right), 309 \mathrm{~nm}$ $\left(17,200 \mathrm{M}^{-1} \mathrm{~cm}^{-1}\right), 289 \mathrm{~nm}\left(\varepsilon=16,200 \mathrm{M}^{-1} \mathrm{~cm}^{-1}\right)$ MS $(\mathrm{EI}=+$ ve mode $)$ : exact mass calculated for $\left[\mathrm{C}_{19} \mathrm{H}_{16} \mathrm{~N}_{4} \mathrm{O}_{2}\right]^{+}: 332.1273$; exact mass found: 332.1267 ; difference: $-2.0 \mathrm{ppm}$.

\section{Tri(ethylferrocene)phosphine Oxide}

In air, tri(ethylferrocene)phosphine $(0.50 \mathrm{~g}, 0.75 \mathrm{mmol})$ was dissolved in THF $(10 \mathrm{~mL})$ in a 50 mL flask. Using a $250 \mu \mathrm{L}$ syringe, a $2.87 \mathrm{M} \mathrm{H}_{2} \mathrm{O}_{2}$ solution $(0.25 \mathrm{~mL}, 0.72 \mathrm{mmol})$ was added to the phosphine solution. After stirring for $3 \mathrm{~h}$, the resulting solution was concentrated in vacuo. The dark orange residue was dissolved in $\mathrm{CHCl}_{3}$ and transferred to a silica gel column $\left(1.5^{\prime \prime} \times\right.$ 8.5"). Using 1:1 hexanes:EtOAc as eluent, tri(ethylferrocene)phosphine $\left(R_{f}=0.66\right)$ was isolated from the column before tri(ethylferrocene)phosphine oxide was eluted using EtOAc $\left(R_{f}=0.54\right)$. The solution containing tri(ethylferrocene)phosphine oxide was concentrated in vacuo to yield an orange solid. Yield $=0.43 \mathrm{~g}, 87 \%$. M.p.: $140-142{ }^{\circ} \mathrm{C} .{ }^{1} \mathrm{H}$ NMR $\left(599.3 \mathrm{MHz}, \mathrm{CDCl}_{3}\right): \delta 4.13(\mathrm{~s}$, $\left.15 \mathrm{H}, \mathrm{C}_{5} \underline{H}_{5}\right), 4.11\left(\mathrm{~s}, 12 \mathrm{H}, \mathrm{C}_{5} \underline{H}_{4} \mathrm{R}\right), 2.71-2.60\left(\mathrm{~m}, 6 \mathrm{H}, \mathrm{C} \underline{H}_{2}\right), 2.00-1.88\left(\mathrm{~m}, 6 \mathrm{H}, \mathrm{C}_{2}\right) .{ }^{13} \mathrm{C}\left\{{ }^{1} \mathrm{H}\right\}$ $\operatorname{NMR}\left(150.7 \mathrm{MHz}, \mathrm{CDCl}_{3}\right): \delta 88.1\left(\mathrm{~d}, J_{\mathrm{CP}}=15 \mathrm{~Hz}\right), 68.6,67.9,67.6,30.1\left(\mathrm{~d}, J_{\mathrm{CP}}=64 \mathrm{~Hz}\right), 22.0$ $\left(\mathrm{d}, J_{\mathrm{CP}}=3 \mathrm{~Hz}\right) \cdot{ }^{31} \mathrm{P}\left\{{ }^{1} \mathrm{H}\right\}$ NMR $\left(242.6 \mathrm{MHz}, \mathrm{CDCl}_{3}\right): \delta 45.6$ (s). FT-IR (ATR): $3082(\mathrm{w}), 2906$ (w), $1444($ w), $1471($ w), $1410($ w), $1323(w), 1226(w), 1212(w), 1191(w), 1170(w), 1159(m)$, $1156(\mathrm{~m}), 1104$ (s), 1045 (w), 998 (s), 941 (w), 921 (7), $810(\mathrm{~s}), 774(\mathrm{~m}), 733(\mathrm{w}), 674(\mathrm{w}), 668$ (w) $\mathrm{cm}^{-1}$. UV-Vis $\left(\mathrm{CH}_{2} \mathrm{Cl}_{2}\right): \lambda_{\max }=438 \mathrm{~nm}\left(\varepsilon=350 \mathrm{M}^{-1} \mathrm{~cm}^{-1}\right)$. Mass Spec. (EI, +ve mode): 
exact mass calculated for $\left[\mathrm{C}_{36} \mathrm{H}_{39} \mathrm{Fe}_{3} \mathrm{OP}\right]^{+}$: 686.0787; exact mass found: 686.0794; difference: +1.0 ppm. Anal. Calcd. (\%) for $\mathrm{C}_{36} \mathrm{H}_{39} \mathrm{Fe}_{3} \mathrm{OP}: \mathrm{C}, 63.01 ; \mathrm{H}$, 5.73. Found: C, 62.70; H, 6.01.

Representative Procedure for the Preparation of Aluminum Formazanate Complexes 7a, $7 b, 8 a$, and 9a.

\section{Complex 7a}

Formazan 6a $(0.39 \mathrm{~g}, 1.2 \mathrm{mmol})$ was dissolved in dry toluene $(40 \mathrm{~mL})$ and stirred for $5 \mathrm{~min}$ before $\mathrm{Al}(\mathrm{O} i \mathrm{Pr})_{3}(0.26 \mathrm{~g}, 1.3 \mathrm{mmol})$ was added. The resulting purple mixture was stirred for 5 min before triphenylphosphine oxide $(0.69 \mathrm{~g}, 2.5 \mathrm{mmol})$ was added and the mixture heated at $120{ }^{\circ} \mathrm{C}$ for $16 \mathrm{~h}$. The colour of the mixture gradually changed from dark purple to green-blue. At this time, the mixture was cooled to $20{ }^{\circ} \mathrm{C}$ and concentrated in vacuo. In air, the dark-purple solid was washed with hexanes $(\sim 50 \mathrm{~mL})$ and collected via vacuum filtration. The crude solid was recrystallized by vapor diffusion of pentane into a saturated $\mathrm{CH}_{2} \mathrm{Cl}_{2}$ solution to afford darkpurple plates. Yield $=0.77$ g, $70 \%$. M.p.: $218-220{ }^{\circ} \mathrm{C} .{ }^{1} \mathrm{H}$ NMR $\left(599.3 \mathrm{MHz}, \mathrm{CDCl}_{3}\right): \delta 8.18(\mathrm{~d}$, $\left.J_{\mathrm{HH}}=8 \mathrm{~Hz}, 2 \mathrm{H}, \operatorname{aryl} \underline{\mathrm{C}} \underline{)}\right), 7.87\left(\mathrm{~d}, J_{\mathrm{HH}}=8 \mathrm{~Hz}, 2 \mathrm{H}, \operatorname{aryl} \underline{\mathrm{C}} \underline{H}\right), 7.54-7.32(\mathrm{~m}, 33 \mathrm{H}, \operatorname{aryl} \underline{\mathrm{C}} \underline{)}), 7.14$ $\left(\mathrm{t}, J_{\mathrm{HH}}=8 \mathrm{~Hz}, 2 \mathrm{H}, \operatorname{aryl} \mathrm{C} \underline{H}\right), 6.96\left(\mathrm{~d}, J_{\mathrm{HH}}=8 \mathrm{~Hz}, 2 \mathrm{H}, \operatorname{aryl} \underline{\mathrm{C}} \underline{)}\right) 6.79\left(\mathrm{t}, J_{\mathrm{HH}}=8 \mathrm{~Hz}, 2 \mathrm{H}, \operatorname{aryl}\right.$ $\mathrm{C} \underline{H}) .{ }^{13} \mathrm{C}\left\{{ }^{1} \mathrm{H}\right\}$ NMR $\left(150.7 \mathrm{MHz}, \mathrm{CDCl}_{3}\right): \delta 157.4,145.5,140.4,138.6,132.8,132.1(\mathrm{~d}$, $\left.J_{\mathrm{CP}}=12 \mathrm{~Hz}\right), 129.3,128.7\left(\mathrm{~d}, J_{\mathrm{CP}}=12 \mathrm{~Hz}\right), 128.2,127.1,125.6,117.5,117.4,114.2 .{ }^{31} \mathrm{P}\left\{{ }^{1} \mathrm{H}\right\}$ NMR (242.6 MHz, $\mathrm{CDCl}_{3}$ ): $\delta$ 35.3. FT-IR (ATR): 3054 (m), 1590 (m), 1472 (m), 1438 (m), 1305 (s), 1242 (s), 1180 (s), 1121 (s), 895 (m), 724 (s), 692 (s), 538 (s) cm ${ }^{-1}$. UV-vis $\left(\mathrm{CH}_{2} \mathrm{Cl}_{2}\right)$ : $\lambda_{\max }=641 \mathrm{~nm}\left(\varepsilon=28,000 \mathrm{M}^{-1} \mathrm{~cm}^{-1}\right), 596 \mathrm{~nm}\left(\varepsilon=21,800 \mathrm{M}^{-1} \mathrm{~cm}^{-1}\right), 329 \mathrm{~nm}\left(\varepsilon=19,300 \mathrm{M}^{-1}\right.$ $\mathrm{cm}^{-1}$; with 50 equiv. of $\mathrm{OPPh}_{3}, \lambda_{\max }=639 \mathrm{~nm}\left(\varepsilon=26,600 \mathrm{M}^{-1} \mathrm{~cm}^{-1}\right), 596 \mathrm{~nm}\left(\varepsilon=20,500 \mathrm{M}^{-1}\right.$ $\left.\mathrm{cm}^{-1}\right), 329 \mathrm{~nm}\left(\varepsilon=16,300 \mathrm{M}^{-1} \mathrm{~cm}^{-1}\right)$. Mass Spec. (ESI, +ve mode): exact mass calculated for 
$\left[\mathrm{C}_{55} \mathrm{H}_{43} \mathrm{AlN}_{4} \mathrm{O}_{4} \mathrm{P}_{2}+\mathrm{Na}\right]^{+}$: 935.2473; exact mass found: 935.2477; difference: +0.4 ppm. Anal. Calcd. (\%) for $\mathrm{C}_{55} \mathrm{H}_{43} \mathrm{AlN}_{4} \mathrm{O}_{4} \mathrm{P}_{2}$ : C, 72.36; H, 4.75; N, 6.14. Found: C, 71.71; H, 5.09; N, 6.17.

\section{Complex 7b}

From formazan $6 \mathbf{b}(0.40 \mathrm{~g}, 1.4 \mathrm{mmol}), \mathrm{Al}(\mathrm{O} i \mathrm{Pr})_{3}(0.52 \mathrm{~g}, 2.6 \mathrm{mmol})$, and triphenylphosphine oxide $(0.85 \mathrm{~g}, 3.1 \mathrm{mmol})$. Yield $=0.88 \mathrm{~g}, 73 \%$ of dark-purple needles. M.p.: $239-241{ }^{\circ} \mathrm{C} .{ }^{1} \mathrm{H}$ NMR (599.3 MHz, $\left.\mathrm{CDCl}_{3}\right): \delta$ 7.54-7.51 (m, 8H, aryl $\left.\mathrm{C} \underline{H}\right), 7.43-7.35(\mathrm{~m}, 24 \mathrm{H}$, aryl $\underline{\mathrm{C}} \underline{\mathrm{H}}), 7.10(\mathrm{t}$, $\left.J_{\mathrm{HH}}=8 \mathrm{~Hz}, 2 \mathrm{H}, \operatorname{aryl~C} \underline{H}\right), 6.82\left(\mathrm{~d}, J_{\mathrm{HH}}=8 \mathrm{~Hz}, 2 \mathrm{H}, \operatorname{aryl} \underline{\mathrm{C}} \underline{)}, 6.65\left(\mathrm{t}, J_{\mathrm{HH}}=8 \mathrm{~Hz}, 2 \mathrm{H}, \operatorname{aryl~C} \underline{H}\right)\right.$. ${ }^{13} \mathrm{C}\left\{{ }^{1} \mathrm{H}\right\} \operatorname{NMR}\left(150.7 \mathrm{MHz}, \mathrm{CDCl}_{3}\right): \delta 159.4,138.6,132.4,132.1\left(\mathrm{~d}, J_{\mathrm{CP}}=10 \mathrm{~Hz}\right), 130.1,129.1$, $128.5\left(\mathrm{~d}, J_{\mathrm{CP}}=13 \mathrm{~Hz}\right), 123.9,120.4,118.5,116.2,114.6 .{ }^{31} \mathrm{P}\left\{{ }^{1} \mathrm{H}\right\}$ NMR $\left(242.6 \mathrm{MHz}, \mathrm{CDCl}_{3}\right): \delta$ 32.3. FT-IR (ATR): 3063 (w), 2921 (w), 2217 (m), 1589 (m), 1471 (m), 1439 (s), 1356 (s), 1305 (s), $1267(\mathrm{~s}), 1258(\mathrm{~s}), 1176(\mathrm{~s}), 1121(\mathrm{~s}), 897$ (w), $745(\mathrm{~m}), 723$ (s), 693 (s), 539 (s) cm $\mathrm{cm}^{-1}$. UVvis $\left(\mathrm{CH}_{2} \mathrm{Cl}_{2}\right): \lambda_{\max }=629 \mathrm{~nm}\left(\varepsilon=24,700 \mathrm{M}^{-1} \mathrm{~cm}^{-1}\right), 586 \mathrm{~nm}\left(\varepsilon=20,700 \mathrm{M}^{-1} \mathrm{~cm}^{-1}\right), 317 \mathrm{~nm}(\varepsilon=$ $\left.8,100 \mathrm{M}^{-1} \mathrm{~cm}^{-1}\right)$; with 50 equiv. of $\mathrm{OPPh}_{3}, \lambda_{\max }=630 \mathrm{~nm}\left(\varepsilon=21,700 \mathrm{M}^{-1} \mathrm{~cm}^{-1}\right), 589 \mathrm{~nm}(\varepsilon=$ $\left.17,700 \mathrm{M}^{-1} \mathrm{~cm}^{-1}\right), 318 \mathrm{~nm}\left(\varepsilon=6,300 \mathrm{M}^{-1} \mathrm{~cm}^{-1}\right)$. Mass Spec. (ESI, +ve mode): exact mass calculated for $\left[\mathrm{C}_{50} \mathrm{H}_{38} \mathrm{AlN}_{5} \mathrm{O}_{4} \mathrm{P}_{2}+\mathrm{Na}\right]^{+}:$884.2112; exact mass found: 884.2118 ; difference: +0.7 ppm. Anal. Calcd. (\%) for $\mathrm{C}_{50} \mathrm{H}_{38} \mathrm{AlN}_{5} \mathrm{O}_{4} \mathrm{P}_{2}: \mathrm{C}, 69.68 ; \mathrm{H}, 4.44 ; \mathrm{N}, 8.13$. Found: $\mathrm{C}, 68.21 ; \mathrm{H}$, $4.57 ; \mathrm{N}, 8.01$.

\section{Complex 8a}

From formazan $6 \mathbf{a}(0.25 \mathrm{~g}, 0.75 \mathrm{mmol}), \mathrm{Al}(\mathrm{OiPr})_{3}(0.18 \mathrm{~g}, 0.88 \mathrm{mmol})$, and tri- $n$-butylphosphine oxide $(0.36 \mathrm{~g}, 1.7 \mathrm{mmol})$. Yield $=0.51 \mathrm{~g}, 86 \%$ of dark-purple plates. M.p.: $>250{ }^{\circ} \mathrm{C} .{ }^{1} \mathrm{H}$ NMR $\left(599.3 \mathrm{MHz}, \mathrm{CDCl}_{3}\right): \delta 8.29\left(\mathrm{~d}, J_{\mathrm{HH}}=8 \mathrm{~Hz}, 2 \mathrm{H}, \operatorname{aryl} \underline{\mathrm{C}} \underline{)}\right) 7.99\left(\mathrm{~d}, J_{\mathrm{HH}}=8 \mathrm{~Hz}, 2 \mathrm{H}\right.$, aryl $\left.\mathrm{C} \underline{H}\right)$, $7.44\left(\mathrm{t}, J_{\mathrm{HH}}=8 \mathrm{~Hz}, 2 \mathrm{H}, \operatorname{aryl} \mathrm{C} \underline{H}\right), 7.32\left(\mathrm{t}, J_{\mathrm{HH}}=7 \mathrm{~Hz}, 1 \mathrm{H}, \operatorname{aryl~C} \underline{H}\right), 7.17\left(\mathrm{t}, J_{\mathrm{HH}}=7 \mathrm{~Hz}, 2 \mathrm{H}, \operatorname{aryl}\right.$ $\mathrm{C} \underline{H}), 7.00\left(\mathrm{~d}, J_{\mathrm{HH}}=8 \mathrm{~Hz}, 2 \mathrm{H}, \operatorname{aryl} \mathrm{C} \underline{H}\right), 6.83\left(\mathrm{t}, J_{\mathrm{HH}}=8 \mathrm{~Hz}, 2 \mathrm{H}\right.$, aryl $\left.\underline{\mathrm{C}} \underline{H}\right), 1.65-1.61(\mathrm{~m}, 12 \mathrm{H}$, 
$\left.\mathrm{C} \underline{H}_{2}\right), 1.35-1.29\left(\mathrm{~m}, 24 \mathrm{H}, \underline{\mathrm{C}}_{2}\right), 0.85-0.83\left(\mathrm{~m}, 18 \mathrm{H}, \mathrm{C}_{3}\right) .{ }^{13} \mathrm{C}\left\{{ }^{1} \mathrm{H}\right\} \mathrm{NMR}\left(150.7 \mathrm{MHz}, \mathrm{CDCl}_{3}\right)$ : $\delta 157.6,145.3,140.5,138.7,129.4,128.3,127.0,125.4,117.6,117.3,114.1,26.3(\mathrm{~d}$, $\left.J_{\mathrm{CP}}=64 \mathrm{~Hz}\right), 24.0\left(\mathrm{~d}, J_{\mathrm{CP}}=15 \mathrm{~Hz}\right), 23.3\left(\mathrm{~d}, J_{\mathrm{CP}}=3 \mathrm{~Hz}\right), 13.4 .{ }^{31} \mathrm{P}\left\{{ }^{1} \mathrm{H}\right\} \mathrm{NMR}(242.6 \mathrm{MHz}$, $\mathrm{CDCl}_{3}$ ): $\delta$ 58.9. FT-IR (ATR): $3058(\mathrm{~m}), 2957$ (s), $2930(\mathrm{~m}), 2870(\mathrm{~m}), 1595$ (m), $1465(\mathrm{~m})$, 1336 (m), 1305 (s), 1243 (s), 1135 (s), 1112 (s), 1019 (m), 896 (m), 765 (m), 741 (s), $578(\mathrm{~s}) \mathrm{cm}^{-1}$. UV-vis $\left(\mathrm{CH}_{2} \mathrm{Cl}_{2}\right): \lambda_{\max }=641 \mathrm{~nm}\left(\varepsilon=33,700 \mathrm{M}^{-1} \mathrm{~cm}^{-1}\right), 596 \mathrm{~nm}\left(\varepsilon=25,100 \mathrm{M}^{-1}\right.$ $\left.\mathrm{cm}^{-1}\right), 329 \mathrm{~nm}\left(\varepsilon=20,000 \mathrm{M}^{-1} \mathrm{~cm}^{-1}\right)$; with 50 equiv. of $\mathrm{OPBu}_{3}, \lambda_{\max }=639 \mathrm{~nm}\left(\varepsilon=31,700 \mathrm{M}^{-1}\right.$ $\left.\mathrm{cm}^{-1}\right), 596 \mathrm{~nm}\left(\varepsilon=24,800 \mathrm{M}^{-1} \mathrm{~cm}^{-1}\right), 329 \mathrm{~nm}\left(\varepsilon=19,400 \mathrm{M}^{-1} \mathrm{~cm}^{-1}\right)$. Mass Spec. (ESI, $+\mathrm{ve}$ mode): exact mass calculated for $\left[\mathrm{C}_{43} \mathrm{H}_{67} \mathrm{AlN}_{4} \mathrm{O}_{4} \mathrm{P}_{2}+\mathrm{Na}\right]^{+}$: 815.4351 ; exact mass found: 815.4346; difference: -0.6 ppm. Anal. Calcd. (\%) for $\mathrm{C}_{43} \mathrm{H}_{67} \mathrm{AlN}_{4} \mathrm{O}_{4} \mathrm{P}_{2}$ : C, 65.13; H, 8.52; N, 7.07. Found: C, $62.84 ; \mathrm{H}, 8.55 ; \mathrm{N}, 6.78$.

\section{Complex 9a}

From formazan $6 \mathbf{6 a}(0.08 \mathrm{~g}, \quad 0.2 \mathrm{mmol}), \quad \mathrm{Al}(\mathrm{O} i \mathrm{Pr})_{3} \quad\left(\begin{array}{lllll}0.07 & \mathrm{~g}, & 0.3 \mathrm{mmol}\end{array}\right)$, and was tri(ethylferrocene)phosphine oxide $(0.34 \mathrm{~g}, 0.50 \mathrm{mmol})$. Yield $=0.18 \mathrm{~g}, 52 \%$ of dark-purple plates. M.p.: $208-210{ }^{\circ} \mathrm{C} .{ }^{1} \mathrm{H}$ NMR $\left(599.3 \mathrm{MHz}, \mathrm{C}_{6} \mathrm{D}_{6}\right): \delta 8.82\left(\mathrm{~d}, J_{\mathrm{HH}}=7 \mathrm{~Hz}, 2 \mathrm{H}\right.$, aryl $\left.\mathrm{C} \underline{H}\right)$, $8.51\left(\mathrm{~d}, J_{\mathrm{HH}}=7 \mathrm{~Hz}, 2 \mathrm{H}, \operatorname{aryl} \mathrm{C} \underline{H}\right), 7.45\left(\mathrm{t}, J_{\mathrm{HH}}=8 \mathrm{~Hz}, 2 \mathrm{H}, \operatorname{aryl} \mathrm{C} \underline{H}\right), 7.37-7.29(\mathrm{~m}, 4 \mathrm{H}, \operatorname{aryl}$ $\mathrm{C} \underline{H}), 7.21-7.19(\mathrm{~m}, 1 \mathrm{H}$, aryl $\mathrm{C} \underline{H}), 6.98\left(\mathrm{t}, J_{\mathrm{HH}}=7 \mathrm{~Hz}, 2 \mathrm{H}, \operatorname{aryl~} \underline{\mathrm{H}} \underline{)}, 4.12\left(\mathrm{~s}, 30 \mathrm{H}, \mathrm{C}_{5} \underline{H}_{5}\right), 3.96(\mathrm{~s}\right.$, $\left.12 \mathrm{H}, \mathrm{C}_{5} \underline{H}_{4} \mathrm{R}\right), 3.94\left(\mathrm{~s}, 12 \mathrm{H}, \mathrm{C}_{5} \underline{H}_{4} \mathrm{R}\right), 2.33-2.26\left(\mathrm{~m}, 12 \mathrm{H}, \mathrm{C} \underline{H}_{2}\right), 1.65-1.58\left(\mathrm{~m}, 12 \mathrm{H}, \mathrm{C}_{2}\right)$. ${ }^{13} \mathrm{C}\left\{{ }^{1} \mathrm{H}\right\}$ NMR (150.7 MHz, $\left.\mathrm{C}_{6} \mathrm{D}_{6}\right): \delta 160.6,143.3,142.1,141.4,129.1,128.7,126.2,125.6$, 118.0, 117.0, 114.9, $88.1\left(\mathrm{~d}, J_{\mathrm{CP}}=15 \mathrm{~Hz}\right), 69.2,68.4,67.9,28.4\left(\mathrm{~d}, J_{\mathrm{CP}}=63 \mathrm{~Hz}\right), 21.7 .{ }^{31} \mathrm{P}\left\{{ }^{1} \mathrm{H}\right\}$ NMR (242.6 MHz, ${ }_{6} \mathrm{D}_{6}$ ): $\delta$ 51.9. FT-IR (ATR): 3092 (m), 2929 (m), 2860 (w), 1589 (m), 1472 (s), $1328(\mathrm{~m}), 1298(\mathrm{~s}), 1241(\mathrm{~s}), 1140(\mathrm{~s}), 1105(\mathrm{~s}), 1000(\mathrm{~m}), 818(\mathrm{~m}), 744(\mathrm{~m}), 682(\mathrm{~m}), 672$ (m), $578(\mathrm{~m}) \mathrm{cm}^{-1}$. UV-vis $\left(\mathrm{CH}_{2} \mathrm{Cl}_{2}\right): \lambda_{\max }=641 \mathrm{~nm}\left(\varepsilon=28,200 \mathrm{M}^{-1} \mathrm{~cm}^{-1}\right), 596 \mathrm{~nm}$ 
$\left(\varepsilon=21,400 \mathrm{M}^{-1} \mathrm{~cm}^{-1}\right), 329 \mathrm{~nm}\left(\varepsilon=18,900 \mathrm{M}^{-1} \mathrm{~cm}^{-1}\right)$. Mass Spec. (ESI, +ve mode): exact mass calculated for $\left[\mathrm{C}_{91} \mathrm{H}_{91} \mathrm{Al}^{54} \mathrm{Fe}_{2}{ }^{56} \mathrm{Fe}_{4} \mathrm{~N}_{4} \mathrm{O}_{4} \mathrm{P}_{2}\right]^{+}$: 1724.2521; exact mass found: 1724.2523; difference: +0.1 ppm. Anal. Calcd. (\%) for $\mathrm{C}_{91} \mathrm{H}_{91} \mathrm{AlFe}_{6} \mathrm{~N}_{4} \mathrm{O}_{4} \mathrm{P}_{2}: \mathrm{C}, 63.22 ; \mathrm{H}, 5.31 ; \mathrm{N}, 3.24$. Found: C, 63.37; H, 5.59; N, 3.18.

\section{RESULTS AND DISCUSSION}

\section{Synthesis and NMR Spectroscopy}

Formazan 6a was prepared by adapting a literature procedure ${ }^{33}$ whilst formazan $\mathbf{6 b}$ has been reported previously. ${ }^{39}$ Their identity and purity were confirmed using ${ }^{1} \mathrm{H},{ }^{13} \mathrm{C}\left\{{ }^{1} \mathrm{H}\right\}$, FT-IR, and UV-vis absorption spectroscopy and mass-spectrometry. The ${ }^{1} \mathrm{H}$ NMR spectrum of formazan $\mathbf{6 a}$ includes a diagnostic NH signal $(\delta=15.65)$ and the corresponding $\mathrm{UV}$-vis absorption spectrum contains a wavelength of maximum absorbance $\left(\lambda_{\max }\right)$ of $558 \mathrm{~nm}$, which is consistent with data obtained for related formazans (Figures S1 and S2). ${ }^{50}$

Aluminum formazanate complexes were obtained by refluxing the parent formazan in dry and degassed toluene with $\mathrm{Al}(\mathrm{O} i \mathrm{Pr})_{3}$ and two equivalents of triphenyl-, tri- $n$-butyl-, or tri(ethylferrocene)phosphine oxide for $16 \mathrm{~h}$ (Scheme 1). The tri(ethylferrocene)phosphine oxide was prepared through careful oxidation of the parent phosphine ${ }^{38}$ using $\mathrm{H}_{2} \mathrm{O}_{2}$ (Figures $\mathrm{S} 3$ and S4).

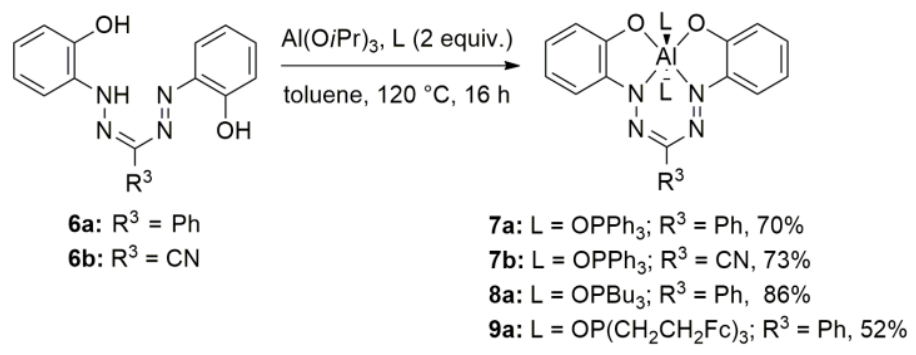

Scheme 1. Synthesis of complexes $7 \mathbf{a}, 7 \mathbf{b}, \mathbf{8 a}$, and $9 \mathbf{a}$. 
Complexes $7 \mathbf{a}, 7 \mathbf{7 b}, \mathbf{8 a}$, and $\mathbf{9 a}$ were isolated in purified yields ranging from 52 to $86 \%$ and characterized using ${ }^{1} \mathrm{H},{ }^{13} \mathrm{C}\left\{{ }^{1} \mathrm{H}\right\},{ }^{31} \mathrm{P}, \mathrm{FT}-\mathrm{IR}$, and UV-vis absorption and PL spectroscopy, cyclic voltammetry, and mass spectrometry (Figures S5-S12). The formation of the phosphineoxide-bound aluminum formazanates was evidenced by a colour change from purple to greenblue and confirmed by the loss of the NH resonance in the respective ${ }^{1} \mathrm{H}$ NMR spectra. In addition, the ${ }^{31} \mathrm{P}\left\{{ }^{1} \mathrm{H}\right\}$ NMR spectra of $7 \mathbf{a}(\delta=35.3), 7 \mathbf{b}(\delta=32.3), 8 \mathbf{a}(\delta=58.9)$, and $9 \mathbf{a}(\delta=$ 51.9) were comprised of a broad singlet shifted downfield relative to the free phosphine oxide. The broad signal is indicative of dynamic ligand exchange at room temperature. In the case of complex $7 \mathbf{a}$, at $-90{ }^{\circ} \mathrm{C}$, three signals were observed; one due to the hexacoordinate complex, one due to free triphenylphosphine oxide, and one due to an intermediate species, likely containing a five-coordinate aluminum atom with a single phosphine oxide donor (Figure S13). Furthermore, the addition of one equivalent of triphenylphosphine oxide to a solution of 7a yielded a ${ }^{31} \mathrm{P}\left\{{ }^{1} \mathrm{H}\right\}$ NMR spectrum comprised of a single broadened resonance at $25{ }^{\circ} \mathrm{C}$ and two signals at $-80{ }^{\circ} \mathrm{C}$; one due to the six-coordinate species and one due to free triphenylphosphine oxide (Figure S14). Given these findings, our solution-phase characterization was conducted in $\mathrm{CH}_{2} \mathrm{Cl}_{2}$ to avoid the competitive binding of polar, coordinating solvents. Furthermore, we studied the solution-based properties both in the presence and absence of excess phosphine oxide to probe the effect of ligand association/dissociation on the physical properties of the complexes.

\section{X-ray Crystallography}

Single crystals of $\mathbf{7 a}, \mathbf{7 b}, \mathbf{8 a}$, and $\mathbf{9 a}$ suitable for $\mathrm{X}$-ray analysis were grown via vapour diffusion of pentane into a saturated $\mathrm{CH}_{2} \mathrm{Cl}_{2}$ solution of the appropriate compound at room temperature (Figure 1, Table 1). The aluminum atoms adopt octahedral geometries in each solid-state structure with the four equatorial sites occupied by the $\mathrm{N}_{2} \mathrm{O}_{2}{ }^{3-}$ formazanate ligand and two phosphine oxide donors coordinated in the axial binding sites. The $\pi$-electrons of the 
formazanate backbones are fully delocalized, as evidenced by the nitrogen-nitrogen and carbonnitrogen bond lengths falling between those associated with single and double bonds of the respective atoms. ${ }^{33}$ The PO bond lengths of 7a [1.4961(5) ̊̊], 7b [1.502(3) ̊̊], 8a [1.5211(11), 1.5171(10) $\AA ; 1.5215(10), 1.5222(11) \AA]^{51}$ and 9a [1.512(3) and 1.497(3) $\left.\AA\right]$ are similar to those of triphenylphosphine oxide aluminum trichloride adducts [1.519(4) $\AA$ ] reported by Burford and co-workers ${ }^{52}$ despite differences in the aluminum ligand field. This suggests that the phosphine oxides are acting as neutral L-type donors. Focusing on the axial Al-O bond lengths of $7 \mathbf{a}$

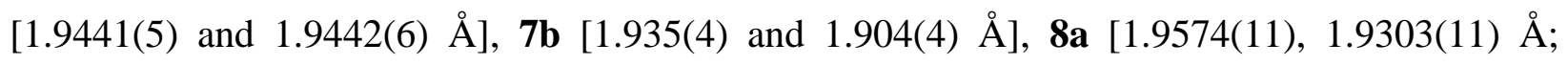
1.9647(12), 1.9252(11) $]$ and 10a [1.903(3) and 1.948(3) $]$, it is evident they are substantially longer than those found in Burford's complexes [Al-O: 1.733(4) $\AA$ ] ${ }^{52}$ but still well within the range of typical Al-O bond lengths $(1.80-2.0 \AA) .{ }^{53} \mathrm{We}$ postulate that the relatively long axial Al$\mathrm{O}$ bonds are a result of steric repulsion between the phosphorus-bound substituents and the formazanate ligand framework. The P-O-Al bond angles of 7a $\left[162.28(4)^{\circ}\right], 7 \mathbf{b}\left[162.3(2)^{\circ}\right.$, $\left.157.1(2)^{\circ}\right], 8 \mathbf{a}\left[136.60(6)^{\circ}, 133.99(6)^{\circ} ; 137.11(7)^{\circ}, 134.65(6)^{\circ}\right]$, and 9a $\left[139.7(2)^{\circ}, 138.4(2)^{\circ}\right]$ also fall within the range observed for other structurally characterized phosphine oxide complexes reported in the literature (P-O-Al: $\left.140-180^{\circ}\right) .{ }^{52,}$ 54-56 The differences in the bond angles observed in complexes supported by aryl phosphine oxides $\mathbf{7 a}$ and $\mathbf{7 b}$ and alkyl phosphine oxides 8a and 9a likely arise due to steric repulsion associated with the phosphine oxide donors and the formazanate ligand backbones. The Al-N and Al-O bonds of the $\mathrm{N}_{2} \mathrm{O}_{2}{ }^{3-}$ binding pocket are similar to those for related structures ${ }^{21}$ and demonstrate that, in all cases, the equatorial Al-O bonds are significantly shorter than the axial Al-O bonds. 

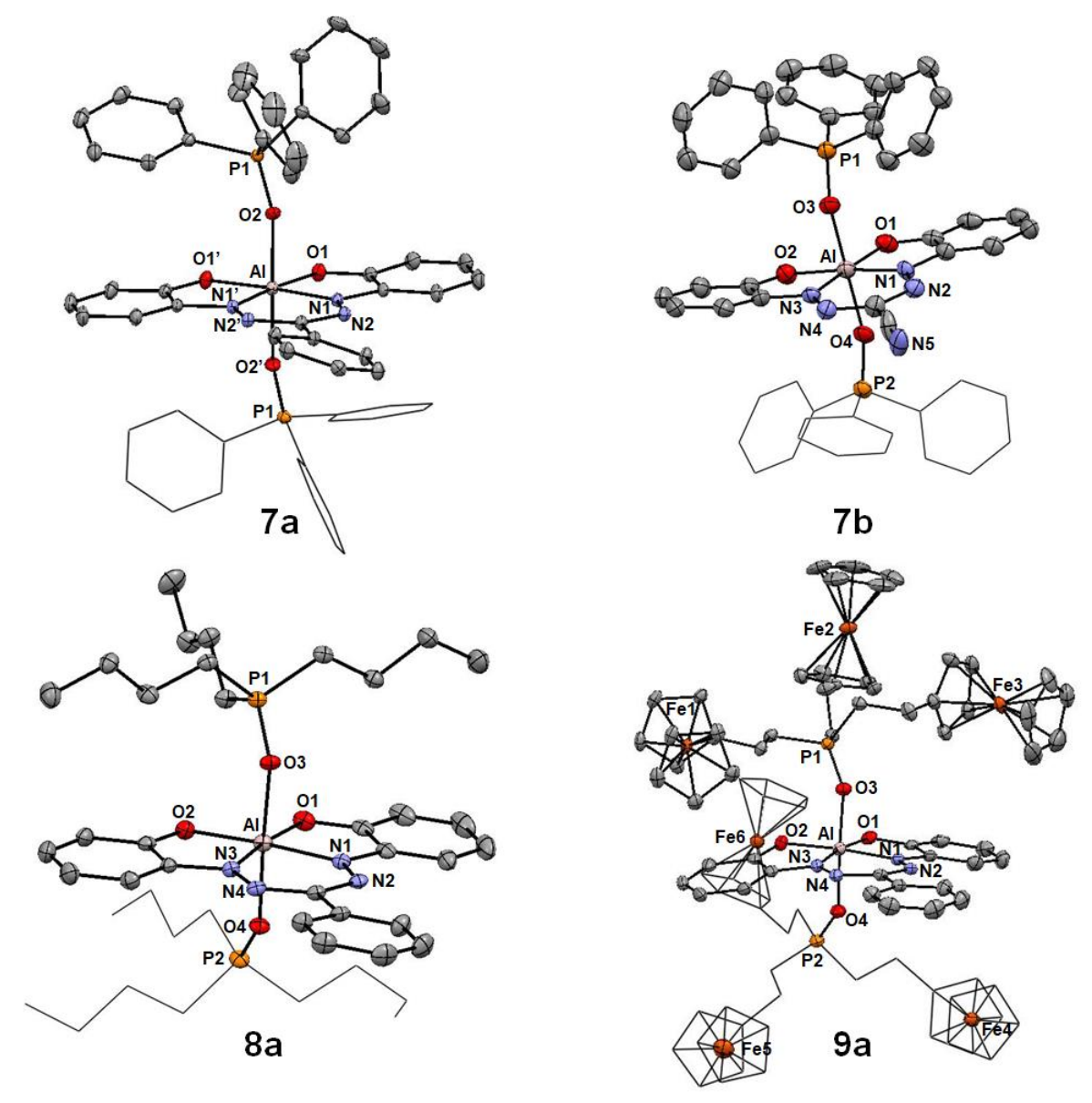

Figure 1. Solid-state structures of complexes 7a, 7b, 8a, and 9a. All anisotropic displacement ellipsoids are shown at $50 \%$ probability. In all cases, one of the axial phosphine oxide donors has been shown as wireframe for clarity. Hydrogen atoms have been omitted for clarity. 
Table 1. Selected bond lengths $(\AA)$ and angles $\left(^{\circ}\right)$ for complexes $\mathbf{7 a}, \mathbf{7 b}, \mathbf{8 a}$, and $9 \mathbf{a}$.

\begin{tabular}{|c|c|c|c|c|c|}
\hline & \multirow[t]{2}{*}{$7 a$} & \multirow[t]{2}{*}{$7 \mathbf{b}$} & \multicolumn{2}{|c|}{$8 \mathbf{a}^{a}$} & \multirow[t]{2}{*}{ 9a } \\
\hline & & & Molecule A & Molecule B & \\
\hline N1-N2 & $1.3023(8)$ & $1.288(5)$ & $1.3060(16)$ & $1.3083(16)$ & $1.304(6)$ \\
\hline N3-N4 & - & $1.299(5)$ & $1.3061(15)$ & $1.3056(15)$ & $1.306(6)$ \\
\hline $\mathrm{C} 1-\mathrm{N} 2$ & $1.3536(6)$ & $1.363(7)$ & $1.3545(18)$ & $1.3558(18)$ & $1.350(6)$ \\
\hline $\mathrm{C} 1-\mathrm{N} 2$, & $1.3536(6)$ & - & - & - & - \\
\hline $\mathrm{C} 1-\mathrm{N} 4$ & - & $1.343(6)$ & $1.3513(18)$ & $1.3560(18)$ & $1.357(6)$ \\
\hline $\mathrm{P} 1-\mathrm{O} 2$ & $1.4961(5)$ & - & - & - & - \\
\hline $\mathrm{P} 1-\mathrm{O} 2$ & $1.4961(5)$ & - & - & - & - \\
\hline $\mathrm{P} 1-\mathrm{O} 3$ & - & $1.502(3)$ & $1.5211(11)$ & $1.5171(10)$ & $1.512(3)$ \\
\hline $\mathrm{P} 2-\mathrm{O} 4$ & - & $1.502(3)$ & $1.5215(10)$ & $1.5222(11)$ & $1.497(3)$ \\
\hline $\mathrm{P} 1-\mathrm{O} 2-\mathrm{Al}$ & $162.28(4)$ & - & - & - & - \\
\hline $\mathrm{P} 1-\mathrm{O} 3-\mathrm{Al}$ & - & $162.3(2)$ & $136.60(6)$ & $133.99(6)$ & $139.7(2)$ \\
\hline $\mathrm{P} 2-\mathrm{O} 4-\mathrm{Al}$ & - & 157.1(2) & $137.11(7)$ & $134.65(6)$ & $138.4(2)$ \\
\hline O1-Al & $1.8314(6)$ & $1.822(4)$ & $1.8381(12)$ & $1.8469(12)$ & $1.846(3)$ \\
\hline $\mathrm{O} 1^{\prime}-\mathrm{Al}$ & $1.8314(6)$ & - & - & - & - \\
\hline $\mathrm{O} 2-\mathrm{Al}$ & $1.9441(5)$ & $1.837(4)$ & $1.8372(12)$ & $1.8444(12)$ & $1.846(3)$ \\
\hline $\mathrm{O} 2^{\prime}-\mathrm{Al}$ & $1.9442(6)$ & - & - & - & - \\
\hline $\mathrm{O} 3-\mathrm{Al}$ & - & $1.935(4)$ & $1.9574(11)$ & $1.9303(11)$ & $1.903(3)$ \\
\hline $\mathrm{O} 4-\mathrm{Al}$ & - & $1.904(4)$ & $1.9647(12)$ & $1.9252(11)$ & $1.948(3)$ \\
\hline N1-Al & $1.9709(6)$ & $1.981(4)$ & $1.9671(14)$ & $1.9698(13)$ & $1.962(4)$ \\
\hline N1'-Al & $1.9710(6)$ & - & - & - & - \\
\hline N3-Al & - & $1.986(4)$ & $1.9683(13)$ & $1.9711(13)$ & $1.968(4)$ \\
\hline N1-Al-O1 & $86.11(3)$ & $85.97(16)$ & $86.12(5)$ & $86.08(5)$ & $86.03(16)$ \\
\hline O1-A1-O2 & $102.89(4)$ & - & $102.95(5)$ & $103.03(5)$ & $102.93(16)$ \\
\hline O2-Al-N3 & $86.11(3)$ & - & $85.76(5)$ & $85.65(5)$ & $85.86(16)$ \\
\hline N3-Al-N1 & $84.89(3)$ & - & $85.18(5)$ & $85.24(5)$ & $85.06(16)$ \\
\hline O1-Al-O1' & - & $103.84(16)$ & - & - & - \\
\hline O1'-Al-N1' & - & $85.22(16)$ & - & - & - \\
\hline $\mathrm{N} 1{ }^{\prime}-\mathrm{Al}-\mathrm{N} 1$ & - & $85.00(16)$ & - & - & - \\
\hline
\end{tabular}

${ }^{a}$ The asymmetric unit determined for complex 8a contains two unique molecules.

\section{UV-vis Absorbance Spectroscopy}

The UV-vis absorption spectra recorded for complexes $\mathbf{7 a}, \mathbf{7 b}, \mathbf{8 a}$, and $9 \mathbf{a}$ in $\mathrm{CH}_{2} \mathrm{Cl}_{2}$ as well as those recorded in the presence of 50 equivalents of phosphine oxide are presented in Figures 2 and S15, and the data are summarized in Table 2. ${ }^{57}$ Each of the complexes studied is strongly absorbing within the UV-vis region of the electromagnetic spectrum. The $\lambda_{\max }$ values observed for the complexes change minimally upon addition of excess phosphine oxide and are red-shifted 
relative to the parent formazans (Figure S17) due to the increased rigidity of the molecule backbone as well as the anionic nature of the ligand. Complex $\mathbf{7 b}$ is luminescent in solution $\left(\lambda_{\mathrm{em}}\right.$ $=690 \mathrm{~nm}, \Phi_{\mathrm{F}}=2 \%$, equation 1) and the intensity of the luminescence reached a maximum when 50 equivalents of $\mathrm{OPPh}_{3}\left(\lambda_{\mathrm{em}}=690 \mathrm{~nm}, \Phi_{\mathrm{F}}=8 \%\right.$, equation 1$)$ were added to the analyte solution. The solution-based equilibria involved in these measurements are heavily shifted toward the sixcoordinate complexes in the presence of excess phosphine oxide. We therefore conclude that it is these complexes that give rise to the optical properties observed.
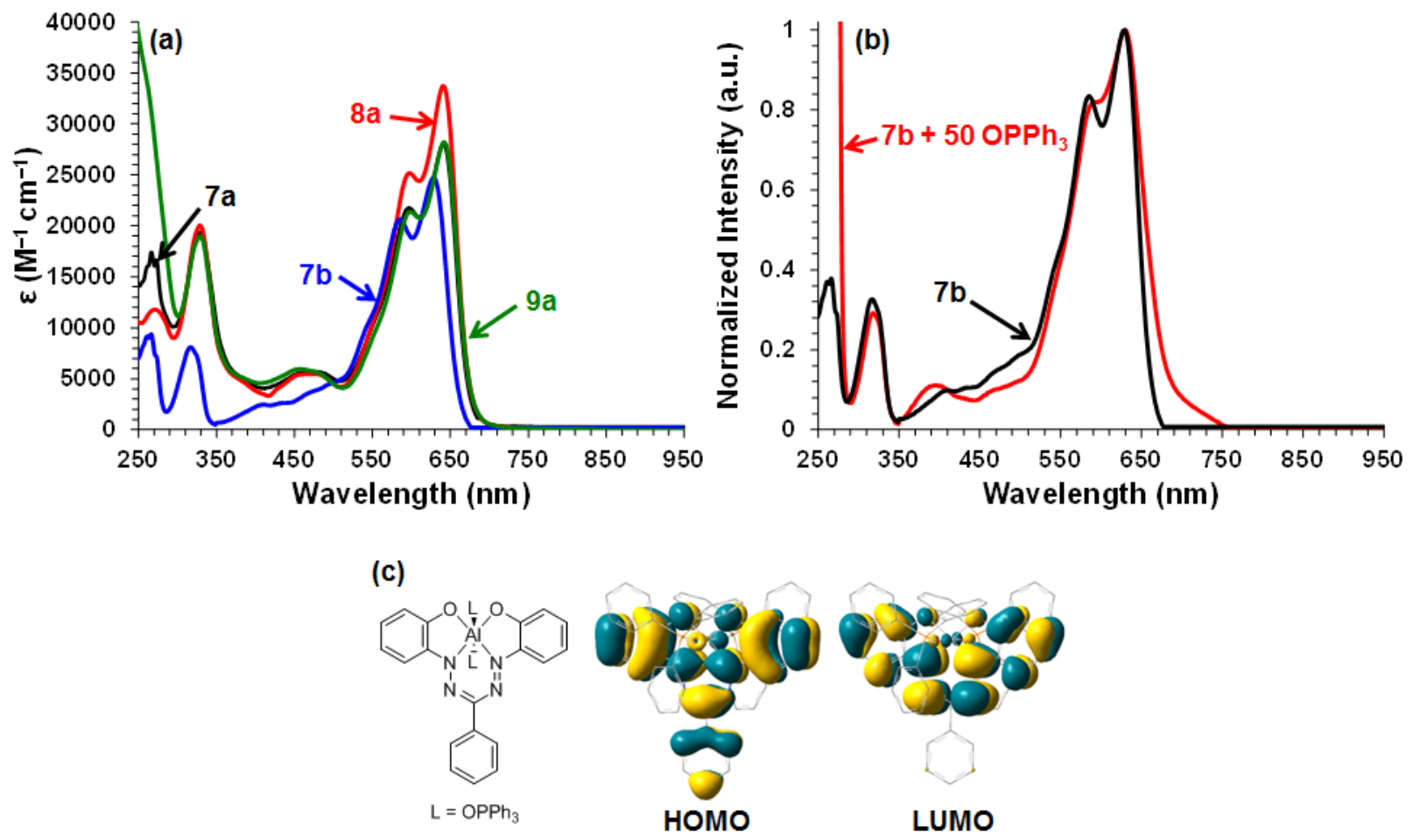

Figure 2. (a) UV-vis absorbance spectra of complexes $7 \mathbf{a}\left(\mathrm{L}=\mathrm{OPPh}_{3}, \mathrm{R}^{3}=\mathrm{Ph}\right), \mathbf{7 b}\left(\mathrm{L}=\mathrm{OPPh}_{3}\right.$, $\left.\mathrm{R}^{3}=\mathrm{CN}\right), \mathbf{8 a}\left(\mathrm{L}=\mathrm{OPBu}_{3}, \mathrm{R}^{3}=\mathrm{Ph}\right), 9 \mathbf{a}\left(\mathrm{L}=\mathrm{OP}\left(\mathrm{CH}_{2} \mathrm{CH}_{2} \mathrm{Fc}\right)_{3}, \mathrm{R}^{3}=\mathrm{Ph}\right)$ recorded for $5 \mu \mathrm{M} \mathrm{CH}_{2} \mathrm{Cl}_{2}$ solutions and (b) normalized UV-vis absorbance spectra of complex $7 \mathbf{b}$ with 0 and 50 equivalents of $\mathrm{OPPh}_{3}$ added. (c) Structure of 7a and its M06/6-311+G(d,p) frontier molecular orbitals computed in $\mathrm{CH}_{2} \mathrm{Cl}_{2}$ solution at the experimental solid-state geometry. 
Table 2. Spectroscopic and electrochemical characterization data for complexes $\mathbf{7 a}, \mathbf{7 b}, \mathbf{8 a}$, and 9a in the absence/presence of excess phosphine oxide.

\begin{tabular}{|c|c|c|c|c|c|}
\hline & $\lambda_{\max }(\mathrm{nm})^{a}$ & $E_{\mathrm{pc}}(\mathrm{V})^{b}$ & $E_{\mathrm{pa} 1}, E_{\mathrm{ox} 1}(\mathrm{~V})^{b, c}$ & $E_{\mathrm{pa} 2,} E_{\mathrm{ox} 2}(\mathrm{~V})^{b, c}$ & $E_{\mathrm{pa} 3}(\mathrm{~V})^{b}$ \\
\hline \multicolumn{6}{|c|}{ O equivalents excess phosphine oxide } \\
\hline \multirow[t]{3}{*}{$7 \mathbf{a}$} & 641 & -1.55 & $0.06,-0.03$ & $0.65,0.61$ & - \\
\hline & 596 & & & & \\
\hline & 329 & & & & \\
\hline \multirow[t]{3}{*}{$7 \mathbf{7 b}$} & 629 & -1.34 & $0.29,0.27$ & $0.97,0.93$ & - \\
\hline & 586 & & & & \\
\hline & 317 & & & & \\
\hline \multirow[t]{3}{*}{$\mathbf{8 a}$} & 641 & -1.54 & $0.03,-0.07$ & $0.65,0.63$ & - \\
\hline & 596 & & & & \\
\hline & 329 & & & & \\
\hline \multirow[t]{3}{*}{ 9a } & 641 & -1.54 & 0.01 & 0.63 & - \\
\hline & 596 & & & & \\
\hline & 329 & & & & \\
\hline \multicolumn{6}{|c|}{50 equivalents excess phosphine oxide } \\
\hline \multirow[t]{3}{*}{$7 \mathbf{a}$} & 639 & -1.75 & -0.03 & 0.62 & 1.08 \\
\hline & 596 & & & & \\
\hline & 329 & & & & \\
\hline \multirow[t]{3}{*}{$7 \mathbf{7 b}$} & 630 & -1.50 & $0.22,0.19$ & 0.88 & 1.08 \\
\hline & 589 & & & & \\
\hline & 318 & & & & \\
\hline \multirow[t]{3}{*}{$8 \mathbf{a}$} & 639 & -1.68 & $-0.12,-0.17$ & 0.65 & 1.10 \\
\hline & 596 & & & & \\
\hline & 329 & & & & \\
\hline
\end{tabular}

${ }^{a}$ Spectra were recorded for $5 \mu \mathrm{M}$ solutions of analyte in dry and degassed $\mathrm{C}_{2} \mathrm{Cl}_{2}$. ${ }^{b} \mathrm{Cyclic}$ voltammetry experiments were conducted in dry and degassed $\mathrm{CH}_{2} \mathrm{Cl}_{2}$ solutions containing $\sim 1$ $\mathrm{mM}$ analyte and $0.1 \mathrm{M}\left[n \mathrm{Bu}_{4}\right]\left[\mathrm{PF}_{6}\right]$ at a scan rate of $0.25 \mathrm{~V} \mathrm{~s}^{-1}$ and internally referenced to the ferrocene/ferrocenium redox couple. ${ }^{c}$ Half wave oxidation potentials $\left(E_{\mathrm{ox} 1}, E_{\mathrm{ox} 2}\right)$ are included for reversible processes.

Aluminum complexes 7a, 8a, and 9a absorb ca. 330, 595, and $640 \mathrm{~nm}$ in $\mathrm{CH}_{2} \mathrm{Cl}_{2}$ indicating that the nature of the phosphine oxide donor $\left[\mathrm{OPPh}_{3}\right.$ vs. $\mathrm{OPBu}_{3}$ vs. $\left.\mathrm{OP}\left(\mathrm{CH}_{2} \mathrm{CH}_{2} \mathrm{Fc}\right)_{3}\right]$ had minimal effect on the spectra collected. The corresponding spectrum recorded for complex 7b was qualitatively similar $\left(\lambda_{\max } c a .318,588,630 \mathrm{~nm}\right)$, although less intense and blue-shifted, due to decreased electronic delocalization associated with the 3-cyano substituent of $\mathbf{7 b}$ compared to the 3-phenyl substituents of 7a, 8a, and 9a. Similar to iron(III) and cobalt(III) 
complexes of $\mathbf{6 b},{ }^{25}$ the spectra of the aluminum(III) complexes recorded in this study each contain two low-energy absorption features between 500 and $700 \mathrm{~nm}$.

To aid interpretation of the UV-vis absorption spectra of 7a, 7b, 8a, and 9a, we used time-dependent density-functional theory (TDDFT) as implemented in the Gaussian 09 $\operatorname{program}^{58}$ to calculate the five lowest electronic excitation energies of one of these complexes, 7a, in $\mathrm{CH}_{2} \mathrm{Cl}_{2}$ solution at the experimental solid-state geometry of the molecule. As a means of reducing the bias associated with the use of one particular methodology, we employed four different density-functional approximations: M06, ${ }^{59}$ M06-2X, ${ }^{59}$ PBE1PBE, ${ }^{60}$ and LC- $\omega$ PBE. ${ }^{61}$ The $6-311+G(d, p)$ basis set was adopted in all cases, and solvation effects were treated implicitly using the polarizable continuum model. These calculations consistently predicted a very strong first absorption at wavelengths ranging from 563 to $636 \mathrm{~nm}$, depending on the functional (see Table S2). We identified this lowest-energy transition with the intense experimental absorption band at $\lambda_{\max }=641 \mathrm{~nm}$. The second-lowest excitation was predicted to occur at a much shorter wavelength ranging between 426 and $507 \mathrm{~nm}$, depending on the functional (Table S2), and had a very low intensity, in stark contrast with the observed spectrum which contains a strong absorption band with a maximum at $596 \mathrm{~nm}$ (Figure 2a,b). The clear absence of a second strong band above $450 \mathrm{~nm}$ in any of the calculated spectra led us to conclude that the observed $\lambda_{\max }=$ $596 \mathrm{~nm}$ band is not a pure electronic excitation but rather a vibronic transition associated with the vibrational excitation of the rigid $\mathrm{N}_{2} \mathrm{O}_{2}{ }^{3-}$ formazanate backbone. The remaining bands observed at $\lambda_{\max }=329 \mathrm{~nm}$ and below appear to correspond to the third-lowest and higher electronic excitations seen in all of the calculated spectra (Table S2).

The calculations also revealed that the highest occupied molecular orbital (HOMO) and the lowest unoccupied molecular orbital (LUMO) were the dominant orbital pair involved in the lowest energy excitation $\left(\pi \rightarrow \pi^{*}\right)$. The HOMO and LUMO isosurfaces (Figure 2c) are indicative 
of highly delocalized electronic structures and closely resemble those of related boron difluoride formazanate complexes, ${ }^{62-63}$ with minor orbital contributions located on the oxygen atoms of the phosphine oxide donors. The lack of frontier orbital density on the phosphorus atoms and their substituents are consistent with the experimental observation that the phosphine oxide donors have a negligible effect on the low-energy absorption maxima of the aluminum complexes described here.

\section{Cyclic Voltammetry}

The electrochemical properties of complexes $\mathbf{7 a}, \mathbf{7 b}, \mathbf{8 a}$, and $9 \mathbf{a}$ were studied using cyclic voltammetry, as were solutions containing 50 equivalents of phosphine oxide (Figures 3, S18 and S19 and Table 2). ${ }^{64}$ The voltammograms recorded were qualitatively similar in the absence/presence of excess phosphine oxide. Each complex gave rise to both reductive and oxidative events within the potential window of $\mathrm{CH}_{2} \mathrm{Cl}_{2}$. Upon scanning to positive potentials, each complex underwent two sequential one-electron oxidations corresponding to the formation of ligand-centred radical cations and dications. The reversibility of these events was affected by the presence of excess phosphine oxide, potentially as a result of poor solution conductivity. Under these conditions, a third oxidation event also emerged at anodic peak potentials $\left(E_{\mathrm{pa}}\right)$ of $1.08-1.10 \mathrm{~V}$, relative to the ferrocene/ferrocenium redox couple. Upon scanning to negative potentials, each complex was reduced electrochemically by one-electron at cathodic peak potentials $\left(E_{\mathrm{pc}}\right)$ between -1.34 and $-1.75 \mathrm{~V}$ to form the corresponding ligand-centred radical anions. The cyclic voltammogram of complex 9a (Figure S19) shared many of the features described above, but was comprised of an intense cathodic peak observed at $c a .-0.12 \mathrm{~V}$. This is likely a result of electrode fouling due to the formation of insoluble species comprised of a formazanate-based cation and up to six ferrocenium cations and stripping upon cathodic scanning. As we are most interested in the six-coordinate complexes in solution, the data 
collected for solutions containing 50 equivalents of phosphine oxide will be discussed below. Changing the $\mathrm{R}^{3}$-substituent from $\mathrm{Ph}$ in $\mathbf{7 a}$ to $\mathrm{CN}$ in $\mathbf{7 b}$ resulted in a shift in the first $\left(\Delta E_{\mathrm{pa1}}\right)$ and second $\left(\Delta E_{\mathrm{pa} 2}\right)$ oxidation events by $0.25 \mathrm{~V}$ and $0.26 \mathrm{~V}$, respectively. This can be attributed to the electron-withdrawing character of the 3-cyano substituent relative to the 3-phenyl substituent. ${ }^{65}$ Altering the axial ligand from triphenylphosphine oxide (7a) to tri- $n$-butylphosphine oxide (8a) had a less dramatic effect on the oxidation potentials observed $\left(\Delta E_{\mathrm{pa} 1}=-0.09 \mathrm{~V}, \Delta E_{\mathrm{pa} 2}=0.03 \mathrm{~V}\right)$. Upon scanning to negative potentials, we observed irreversible one-electron reductions for each complex, likely associated with the formation of a ligand-centred radical anion. Substituent variation at the 3-position in $7 \mathbf{a}$ and $7 \mathbf{b}$ resulted in a $\Delta E_{\mathrm{pc}}$ of $0.25 \mathrm{~V}$. The nature of the axial ligand had minimal effect on the reduction potentials observed $\left(\Delta E_{\mathrm{pc}}=0.07 \mathrm{~V}\right)$.
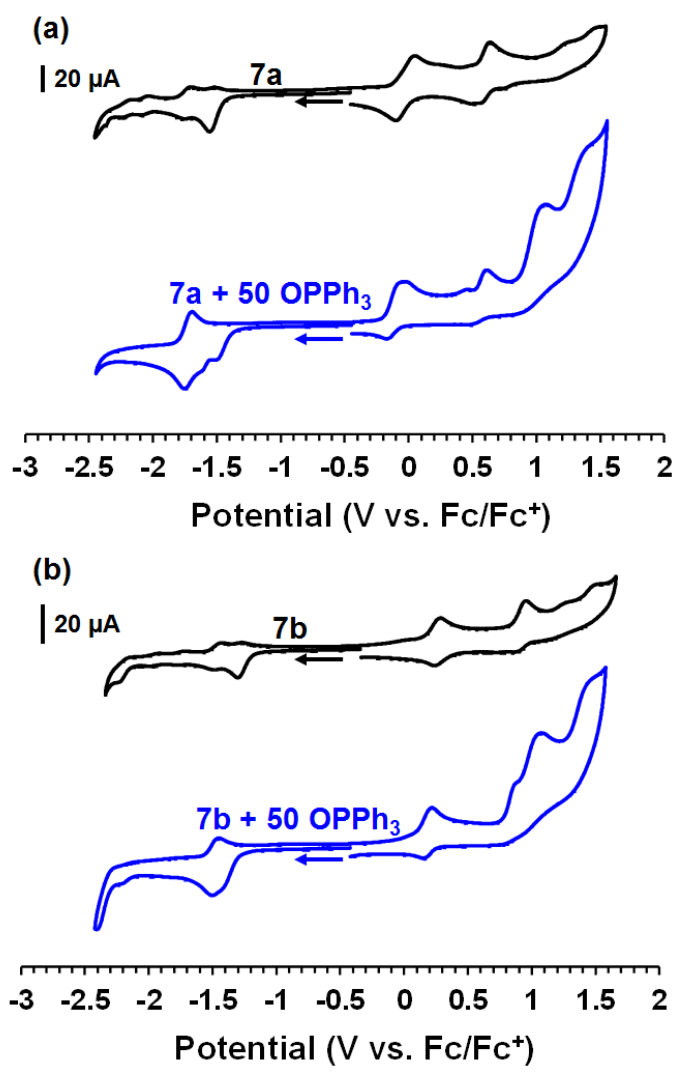

Figure 3. Cyclic voltammograms recorded for (a) complex 7a $\left(\mathrm{L}=\mathrm{OPPh}_{3}, \mathrm{R}^{3}=\mathrm{Ph}\right)$ and (b) complex $7 \mathbf{b}\left(\mathrm{L}=\mathrm{OPPh}_{3}, \mathrm{R}^{3}=\mathrm{CN}\right.$ in degassed $\mathrm{CH}_{2} \mathrm{Cl}_{2}$ containing $\sim 1 \mathrm{mM}$ analyte and $0.1 \mathrm{M}$ $\left[n \mathrm{Bu}_{4} \mathrm{~N}\right]\left[\mathrm{PF}_{6}\right]$ at a scan rate of $0.25 \mathrm{~V} \mathrm{~s}^{-1}$ with 0 (black lines) and 50 equivalents $\mathrm{OPPh}_{3}$ (blue lines). The arrows denote the scan direction. 


\section{Electrochemiluminescence Studies}

Based on the unique PL and electrochemical properties of complex $\mathbf{7 b}$ in the presence of 50 equivalents of $\mathrm{OPPh}_{3}$, we conducted ECL studies. In the absence of a coreactant, ECL was not detected between -2.25 and $1.25 \mathrm{~V}$, relative to the ferrocene/ferrocenium redox couple. Figure 4a displays the cyclic voltammogram along with the corresponding ECL-voltage curve for $7 \mathbf{b}$ in the presence of $5 \mathrm{mM}$ TPrA. The introduction of a coreactant often results in enhanced ECL, as the coreactant and analyte-based radicals required to produce the excited state exist at similar potentials. The electrogenerated radical $\operatorname{TPrA}^{\bullet}$ has emerged as a useful coreactant for the enhancement of ECL. The first ECL peak observed for $\mathbf{7 b}$ in the ECL-voltage curve appears at $0.51 \mathrm{~V}$, a potential at which $\mathbf{7 b}^{+\bullet}$ and $\operatorname{TPr} \mathrm{A}^{\bullet}$ coexist. ${ }^{42,66}$ When these species meet in the vicinity of the working electrode, we postulate that a single electron is transferred from $\operatorname{TPr} A^{\bullet}$ to $\mathbf{7 b}^{+\bullet}$, generating an emissive excited state $\left(\mathbf{7} \mathbf{b}^{*}\right)$.

When scanning towards more positive potentials, a second ECL maximum was observed at a potential of $0.86 \mathrm{~V}$, whereby $\mathbf{7} \mathbf{b}^{2+}$ was produced in the vicinity of the working electrode. Based on conclusions drawn from detailed studies of related $\mathrm{BF}_{2}$ adducts of formazanates, ${ }^{36}$ we postulate that the dication is implicated in a comproportionation reaction $\left(\mathbf{7 b}^{\mathbf{2 +}}+\mathbf{7 b} \rightarrow 2 \mathbf{b} \mathbf{b}^{+\bullet}\right)$, ultimately resulting in the production of $\mathbf{7 b}^{+\boldsymbol{\bullet}}$ in the vicinity of the working electrode that can further react with $\operatorname{TPrA}^{*}$ to produce the emissive excited state $\mathbf{7 b ^ { * }}$. The relatively low ECL intensity at this potential is a reflection of the poor stability of dication $\mathbf{7} \mathbf{b}^{\mathbf{2 +}}$ in solution. Upon scanning the potential in the reverse direction, the ECL intensity decreased due to reduced concentrations of $\operatorname{TPrA}^{\bullet}$ and $\mathbf{7 b}^{+\bullet}$. The ECL efficiency of the $7 \mathbf{b} / \mathrm{TPr}$ A system was calculated to be $7 \%$ relative to the $\left[\mathrm{Ru}(\mathrm{bpy})_{3}\right]\left[\mathrm{PF}_{6}\right]_{2} / \mathrm{TPrA}$ system under identical conditions using equation 2 .

The ECL of the 7b/TPrA system was further studied with ECL spectroscopy. The PL spectrum of $\mathbf{7 b}$ revealed a maximum intensity at $690 \mathrm{~nm}$, while the ECL accumulated spectrum 
obtained in the course of two consecutive potential scan cycles between $-0.24 \mathrm{~V}$ and $1.26 \mathrm{~V}$ had maximum intensity at a wavelength of $735 \mathrm{~nm}$ (Figure 4b). The $45 \mathrm{~nm}$ difference between the PL and ECL maxima is likely a result of the inner-filter effect caused by self-absorption, ${ }^{67}$ but we cannot conclusively rule out excimer formation ${ }^{44,68}$ as boron difluoride complexes of formazanate ligands have been shown to aggregate via a number of different $\pi$-type interactions during studies of their aggregation-induced emission. ${ }^{69}$

Spooling ECL spectroscopy is an ideal technique to monitor in real time the evolution and devolution of ECL during the scanning of applied potentials. ${ }^{70}$ As shown in Figure $4 \mathrm{c}$, the ECL onset potential was found at $t=10 \mathrm{~s}(0.26 \mathrm{~V})$, and the ECL spectra showed a peak wavelength of $735 \mathrm{~nm}$. The ECL emission intensity reached its maximum intensity at $t=15 \mathrm{~s}$ $(0.51 \mathrm{~V})$. The ECL evolution and devolution patterns demonstrated by spooling spectroscopy agree well with the ECL-voltage curve exhibited in Figure 4a. Furthermore, Figure 4d and 4e demonstrate that there is no shift in the ECL peak wavelength during the potential scanning, indicating that there is a single excited state implicated during ECL evolution and devolution. This conclusion agrees well with the ECL mechanisms described above. 

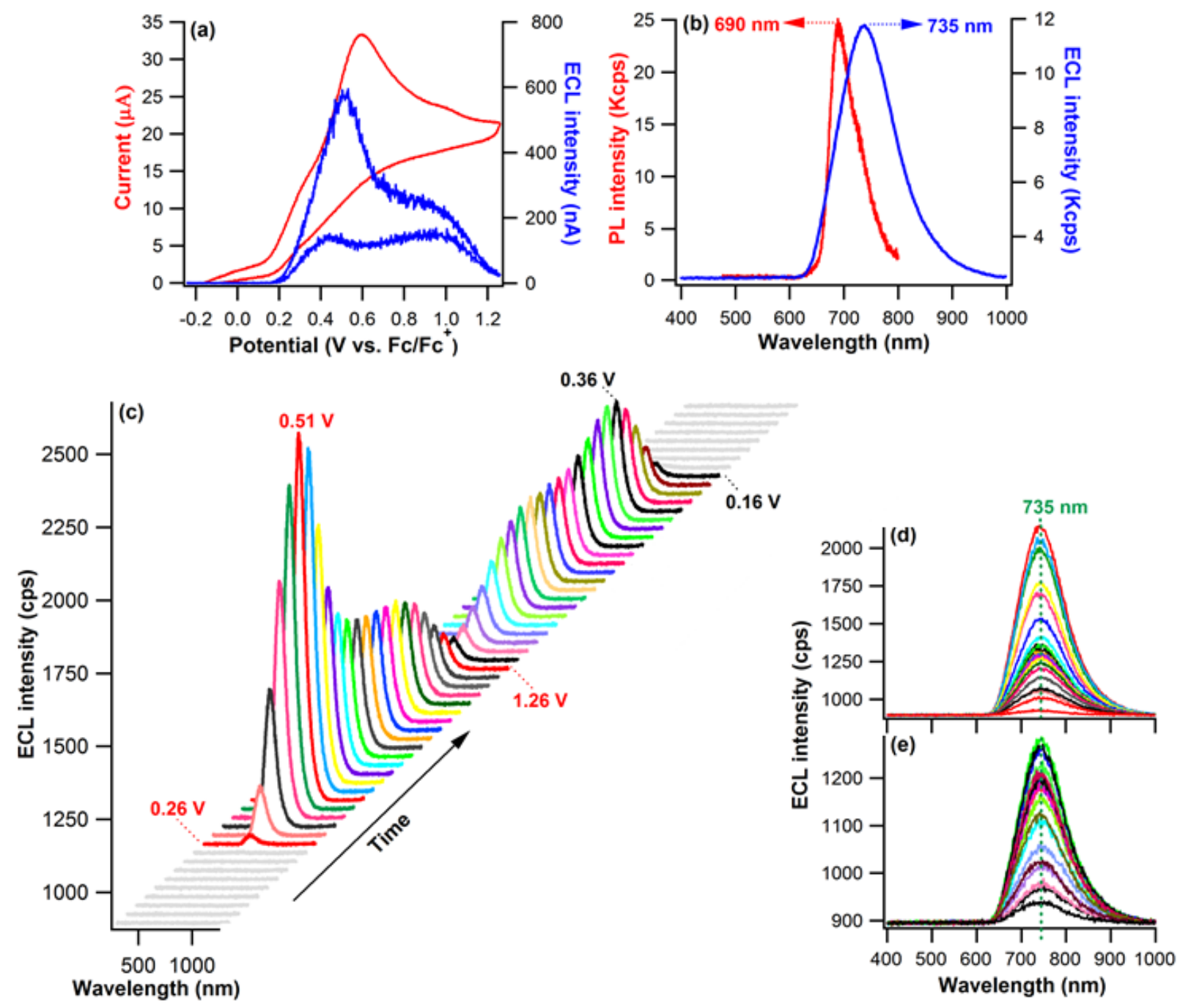

Figure 4. (a) Cyclic voltammogram (red) and ECL-voltage curve (blue) collected for a $\mathrm{CH}_{2} \mathrm{Cl}_{2}$ solution containing $0.6 \mathrm{mM}$ of complex $7 \mathbf{b}, 5 \mathrm{mM}$ TPrA, $30 \mathrm{mM} \mathrm{OPPh}$, and $0.1 \mathrm{M}$ $\left[n \mathrm{Bu}_{4} \mathrm{~N}\right]\left[\mathrm{PF}_{6}\right]$ as supporting electrolyte. (b) Photoluminescence spectrum of complex $7 \mathbf{b}$ (red) and accumulated ECL spectrum of complex 7b/TPrA (blue) in $\mathrm{CH}_{2} \mathrm{Cl}_{2}$. (c) Spooled ECL spectra, (d) evolution, and (e) devolution spectra collected for a $\mathrm{CH}_{2} \mathrm{Cl}_{2}$ solution containing $0.6 \mathrm{mM}$ of complex 7b, $5 \mathrm{mM}$ TPrA, $30 \mathrm{mM} \mathrm{OPPh}_{3}$, and $0.1 \mathrm{M}\left[n \mathrm{Bu}_{4} \mathrm{~N}\right]\left[\mathrm{PF}_{6}\right]$ as supporting electrolyte acquired by reversibly scanning between $-0.24 \mathrm{~V}$ and $1.26 \mathrm{~V}$ at a scan rate of $0.025 \mathrm{~V} \mathrm{~s}^{-1}$ and a time interval of $2 \mathrm{~s}$ for each spectrum.

\section{CONCLUSIONS}

We have synthesized a new family of aluminum(III) complexes of $\mathrm{N}_{2} \mathrm{O}_{2}{ }^{3-}$ formazanate ligands supported by L-type phosphine oxide donors. The aluminum atoms in these complexes exist in octahedral geometries in the solid state, with the formazanate ligands occupying the four 
equatorial coordination sites and two phosphine oxide donors occupying the axial sites. Each of the complexes can be oxidized or reduced electrochemically, as evidenced by cyclic voltammetry experiments; replacing the 3-phenyl substituent with an electron-withdrawing 3cyano substituent shifted both the reduction and oxidation potentials to more positive values, whereas the nature of the axial phosphine oxide had a less dramatic effect. Similarly, the identity of the axial phosphine oxide had minimal influence over the observed UV-vis absorbance maxima, whereas the introduction of a 3-cyano substituent resulted in a blue-shift in $\lambda_{\max }$. TDDFT calculations revealed that the HOMO and LUMO of the respective compounds were the dominant orbital pair involved in the lowest-energy transition. We also investigated the solutionbased equilibria associated with phosphine-oxide ligation/dissocation and confirmed that the emission intensity of the six-coordinate complex $\mathbf{7 b}$ was maximized in the presence of excess ligand. The unique electrochemical and PL properties of $\mathbf{7 b}$ prompted us to study its electrochemiluminescence properties in the presence of tri-n-propylamine and excess $\mathrm{OPPh}_{3}$, revealing an ECL maximum that was red-shifted by $c a .45 \mathrm{~nm}$ relative to the PL maximum wavelength recorded independently. These studies have demonstrated the potential of sixcoordinate aluminum formazanate complexes as functional materials.

\section{ASSOCIATED CONTENT}

\section{Supporting Information}

The supporting information is available free of charge on the ACS Publications website at DOI: \#\#\#\#\#\#.

X-ray diffraction data collection and refinement details; ${ }^{1} \mathrm{H},{ }^{13} \mathrm{C}\left\{{ }^{1} \mathrm{H}\right\}$, and ${ }^{31} \mathrm{P}$ NMR spectra; UVvis absorption spectra; cyclic voltammograms; and computational details.

\section{AUTHOR INFORMATION}




\section{Corresponding Authors}

*E-mail: joe.gilroy@uwo.ca. Tel: +1 (519) 661-2111 ext. 81561.

\section{Author Contributions}

The manuscript was written through contributions of all authors. All authors have given approval of the final version of the manuscript.

\section{Notes}

The authors declare no competing financial interests.

\section{ACKNOWLEDGEMENTS}

This work was supported by the Natural Sciences and Engineering Research Council (NSERC) of Canada (J.B.G.: DG RGPIN-2013-435675, V.N.S.: DG RGPIN-2015-04814, Z.D.: DG RGPIN-2013-201697 and SPG STPGP-2016-493924 and R.R.M.: CGS-D Scholarship), the Ontario Ministry of Research and Innovation (J.B.G.: ERA, ER-14-10-147) and the Canadian Foundation for Innovation (J.B.G.: JELF, 33977).

\section{REFERENCES}

(1) Sarazin, Y.; Carpentier, J.-F. Discrete Cationic Complexes for Ring-Opening Polymerization Catalysis of Cyclic Esters and Epoxides. Chem. Rev. 2015, 115, 3564-3614.

(2) Atwood, D. A.; Jegier, J. A.; Rutherford, D. Cationic Aluminum Compounds with Potential Relevance to Lewis Acid Catalysis. J. Am. Chem. Soc. 1995, 117, 6779-6780.

(3) Whiteoak, C. J.; Kielland, N.; Laserna, V.; Escudero-Adán, E. C.; Martin, E.; Kleij, A. W. A Powerful Aluminum Catalyst for the Synthesis of Highly Functional Organic Carbonates. J. Am. Chem. Soc. 2013, 135, 1228-1231. 
(4) North, M.; Quek, S. C. Z.; Pridmore, N. E.; Whitwood, A. C.; Wu, X. Aluminum(salen) Complexes as Catalysts for the Kinetic Resolution of Terminal Epoxides via $\mathrm{CO}_{2}$ Coupling. ACS Catal. 2015, 5, 3398-3402.

(5) Berben, L. A. Catalysis by Aluminum(III) Complexes of Non-Innocent Ligands. Chem. Eur. J. 2015, 21, 2734-2742.

(6) Wang, S. Luminescence and Electroluminescence of $\mathrm{Al}(\mathrm{III}), \mathrm{B}(\mathrm{III}), \mathrm{Be}(\mathrm{II})$ and $\mathrm{Zn}(\mathrm{II})$ Complexes with Nitrogen Donors. Coord. Chem. Rev. 2001, 215, 79-98.

(7) Tang, C. W.; VanSlyke, S. A. Organic Electroluminescent Diodes. Appl. Phys. Lett. 1987, $51,913-915$.

(8) Liao, S.-H.; Shiu, J.-R.; Liu, S.-W.; Yeh, S.-J.; Chen, Y.-H.; Chen, C.-T.; Chow, T. J.; Wu, C.-I. Hydroxynaphthyridine-Derived Group III Metal Chelates: Wide Band Gap and Deep Blue Analogues of Green $\mathrm{Alq}_{3}$ (Tris(8-hydroxyquinolate)aluminum) and Their Versatile Applications for Organic Light-Emitting Diodes. J. Am. Chem. Soc. 2009, 131, 763-777.

(9) Pérez-Bolívar, C.; Takizawa, S.-y.; Nishimura, G.; Montes, V. A.; Anzenbacher, P. HighEfficiency Tris(8-hydroxyquinoline)aluminum ( $\left.\mathrm{Alq}_{3}\right)$ Complexes for Organic White-LightEmitting Diodes and Solid-State Lighting. Chem. Eur. J. 2011, 17, 9076-9082.

(10) Yaroshevsky, A. A. Abundances of Chemical Elements in the Earth's Crust. Geochem. Int. 2006, 44, 48-55.

(11) Li, J.; Zhang, K.; Huang, H.; Yu, A.; Hu, H.; Cui, H.; Cui, C. Synthesis and Reactions of a Redox-Active $\alpha$-Diimine Aluminum Complex. Organometallics 2013, 32, 1630-1635.

(12) Cole, B. E.; Wolbach, J. P.; Dougherty, W. G.; Piro, N. A.; Kassel, W. S.; Graves, C. R. Synthesis and Characterization of Aluminum- $\alpha$-Diimine Complexes over Multiple Redox States. Inorg. Chem. 2014, 53, 3899-3906. 
(13) Cross, E. D.; Allan, L. E. N.; Decken, A.; Shaver, M. P. Aluminum Salen and Salan Complexes in the Ring-Opening Polymerization of Cyclic Esters: Controlled Immortal and Copolymerization of rac- $\beta$-Butyrolactone and rac-Lactide. J. Polym. Sci., Part A: Polym. Chem. 2013, 51, 1137-1146.

(14) Pepels, M. P. F.; Bouyahyi, M.; Heise, A.; Duchateau, R. Kinetic Investigation on the Catalytic Ring-Opening (Co)Polymerization of (Macro)Lactones Using Aluminum Salen Catalysts. Macromolecules 2013, 46, 4324-4334.

(15) Myers, T. W.; Kazem, N.; Stoll, S.; Britt, R. D.; Shanmugam, M.; Berben, L. A. A Redox Series of Aluminum Complexes: Characterization of Four Oxidation States Including a Ligand Biradical State Stabilized via Exchange Coupling. J. Am. Chem. Soc. 2011, 133, 8662-8672.

(16) Myers, T. W.; Berben, L. A. Aluminum-Ligand Cooperative $\mathrm{N}-\mathrm{H}$ Bond Activation and an Example of Dehydrogenative Coupling. J. Am. Chem. Soc. 2013, 135, 9988-9990.

(17) Baudron, S. A. Luminescent Dipyrrin Based Metal Complexes. Dalton Trans. 2013, 42, 7498-7509.

(18) Ikeda, C.; Ueda, S.; Nabeshima, T. Aluminium Complexes of $\mathrm{N}_{2} \mathrm{O}_{2}$-Type Dipyrrins: The First Hetero-Multinuclear Complexes of Metallo-Dipyrrins with High Fluorescence Quantum Yields. Chem. Commun. 2009, 2544-2546.

(19) Thompson, E. J.; Berben, L. A. Electrocatalytic Hydrogen Production by an Aluminum(III) Complex: Ligand-Based Proton and Electron Transfer. Angew. Chem. Int. Ed. 2015, 54, 1164211646.

(20) Van Zee, N. J.; Sanford, M. J.; Coates, G. W. Electronic Effects of Aluminum Complexes in the Copolymerization of Propylene Oxide with Tricyclic Anhydrides: Access to Well-Defined, Functionalizable Aliphatic Polyesters. J. Am. Chem. Soc. 2016, 138, 2755-2761. 
(21) Saikawa, M.; Daicho, M.; Nakamura, T.; Uchida, J.; Yamamura, M.; Nabeshima, T. Synthesis of a New Family of Ionophores Based on Aluminum-Dipyrrin Complexes (ALDIPYs) and their Strong Recognition of Alkaline Earth Ions. Chem. Commun. 2016, 52, 4014-4017.

(22) Gong, S.; Ma, H. $\beta$-Diketiminate Aluminium Complexes: Synthesis, Characterization and Ring-Opening Polymerization of Cyclic Esters. Dalton Trans. 2008, 3345-3357.

(23) Bourget-Merle, L.; Lappert, M. F.; Severn, J. R. The Chemistry of $\beta$-Diketiminatometal Complexes. Chem. Rev. 2002, 102, 3031-3066.

(24) Sigeikin, G. I.; Lipunova, G. N.; Pervova, I. G. Formazans and their Metal Complexes. Russ. Chem. Rev. 2006, 75, 885-900.

(25) Gilroy, J. B.; Patrick, B. O.; McDonald, R.; Hicks, R. G. Transition Metal Complexes of 3Cyano- and 3-Nitroformazans. Inorg. Chem. 2008, 47, 1287-1294.

(26) Hong, S.; Hill, L. M. R.; Gupta, A. K.; Naab, B. D.; Gilroy, J. B.; Hicks, R. G.; Cramer, C. J.; Tolman, W. B. Effects of Electron-Deficient $\beta$-Diketiminate and Formazan Supporting Ligands on Copper(I)-Mediated Dioxygen Activation. Inorg. Chem. 2009, 48, 4514-4523.

(27) Mandal, A.; Schwederski, B.; Fiedler, J.; Kaim, W.; Lahiri, G. K. Evidence for Bidirectional Noninnocent Behavior of a Formazanate Ligand in Ruthenium Complexes. Inorg. Chem. 2015, 54, 8126-8135.

(28) Kabir, E.; Wu, C.-H.; Wu, J. I. C.; Teets, T. S. Heteroleptic Complexes of Cyclometalated Platinum with Triarylformazanate Ligands. Inorg. Chem. 2016, 55, 956-963.

(29) Protasenko, N. A.; Poddel'sky, A. I.; Bogomyakov, A. S.; Fukin, G. K.; Cherkasov, V. K. Heteroligand o-Semiquinonato-Formazanato Cobalt Complexes. Inorg. Chem. 2015, 54, 60786080 . 
(30) Travieso-Puente, R.; Broekman, J. O. P.; Chang, M.-C.; Demeshko, S.; Meyer, F.; Otten, E. Spin-Crossover in a Pseudo-tetrahedral Bis(formazanate) Iron Complex. J. Am. Chem. Soc. 2016, 138, 5503-5506.

(31) Barbon, S. M.; Reinkeluers, P. A.; Price, J. T.; Staroverov, V. N.; Gilroy, J. B. Structurally Tunable 3-Cyanoformazanate Boron Difluoride Dyes. Chem. Eur. J. 2014, 20, 11340-11344.

(32) Chang, M.-C.; Dann, T.; Day, D. P.; Lutz, M.; Wildgoose, G. G.; Otten, E. The Formazanate Ligand as an Electron Reservoir: Bis(Formazanate) Zinc Complexes Isolated in Three Redox States. Angew. Chem. Int. Ed. 2014, 53, 4118-4122.

(33) Maar, R. R.; Barbon, S. M.; Sharma, N.; Groom, H.; Luyt, L. G.; Gilroy, J. B. Evaluation of Anisole-Substituted Boron Difluoride Formazanate Complexes for Fluorescence Cell Imaging. Chem. Eur. J. 2015, 21, 15589-15599.

(34) Chang, M.-C.; Otten, E. Intramolecular Hydride Transfer Reactions in (Formazanate)Boron Dihydride Complexes. Organometallics 2016, 35, 534-542.

(35) Barbon, S. M.; Staroverov, V. N.; Gilroy, J. B. Structurally Diverse Boron-Nitrogen Heterocycles from an $\mathrm{N}_{2} \mathrm{O}_{2}{ }^{3-}$ Formazanate Ligand. Angew. Chem. Int. Ed. 2017, 56, 8173-8177. (36) Hesari, M.; Barbon, S. M.; Staroverov, V. N.; Ding, Z.; Gilroy, J. B. Efficient Electrochemiluminescence of a Readily Accessible Boron Difluoride Formazanate Dye. Chem. Commun. 2015, 51, 3766-3769.

(37) Schorn, W.; Grosse-Hagenbrock, D.; Oelkers, B.; Sundermeyer, J. Formazanido Complexes of Heavier Group 13 Elements Aluminium, Gallium, and Indium. Dalton Trans. 2016, 45, 12011207.

(38) Rabiee Kenaree, A.; Berven, B. M.; Ragogna, P. J.; Gilroy, J. B. Highly-Metallized Phosphonium Polyelectrolytes. Chem. Commun. 2014, 50, 10714-10717. 
(39) Gilroy, J. B.; Otieno, P. O.; Ferguson, M. J.; McDonald, R.; Hicks, R. G. Synthesis and Characterization of 3-Cyano- and 3-Nitroformazans, Nitrogen-Rich Analogues of $\beta$-Diketimine Ligands. Inorg. Chem. 2008, 47, 1279-1286.

(40) Suzuki, K.; Kobayashi, A.; Kaneko, S.; Takehira, K.; Yoshihara, T.; Ishida, H.; Shiina, Y.; Oishi, S.; Tobita, S. Reevaluation of Absolute Luminescence Quantum Yields of Standard Solutions Using a Spectrometer with an Integrating Sphere and a Back-Thinned CCD Detector. Phys. Chem. Chem. Phys. 2009, 11, 9850-9860.

(41) McCord, P.; Bard, A. J. Electrogenerated Chemiluminescence: Part 54. Electrogenerated Chemiluminescence of Ruthenium(II) 4,4'-Diphenyl-2,2'-Bipyridine and Ruthenium(II) 4,7Diphenyl-1,10-Phenanthroline Systems in Aqueous and Acetonitrile Solutions. J. Electroanal. Chem. 1991, 318, 91-99.

(42) Lai, R. Y.; Bard, A. J. Electrogenerated Chemiluminescence. 70. The Application of ECL to Determine Electrode Potentials of Tri-n-propylamine, Its Radical Cation, and Intermediate Free Radical in MeCN/Benzene Solutions. J. Phys. Chem. A 2003, 107, 3335-3340.

(43) Richter, M. M. Electrochemiluminescence (ECL). Chem. Rev. 2004, 104, 3003-3036.

(44) Hesari, M.; Lu, J.-s.; Wang, S.; Ding, Z. Efficient Electrochemiluminescence of a BoronDipyrromethene (BODIPY) Dye. Chem. Commun. 2015, 51, 1081-1084.

(45) Bruker-AXS, SAINT version 2013.8, 2013, Bruker-AXS Madison, WI 53711, USA.

(46) Bruker-AXS, SADABS version 2012.1, 2012, Bruker-AXS, Madison, WI 53711, USA.

(47) Sheldrick, G. M. SHELXT-Integrated Space-Group and Crystal-Structure Determination. Acta Cryst. 2015, A71, 3-8.

(48) Spek, A. L. PLATON SQUEEZE: A Tool for the Calculation of the Disordered Solvent Contribution to the Calculated Structure Factors. Acta Cryst. 2015, C71, 9-18.

(49) Sheldrick, G. M. A Short History of SHELX. Acta Cryst. 2008, A64, 112-122. 
(50) Barbon, S. M.; Price, J. T.; Reinkeluers, P. A.; Gilroy, J. B. Substituent-Dependent Optical and Electrochemical Properties of Triarylformazanate Boron Difluoride Complexes. Inorg. Chem. 2014, 53, 10585-10593.

(51) The asymmetric unit determined for complex 8a contains two unique molecules.

(52) Burford, N.; Royan, B. W.; Spence, R. E. v. H.; Cameron, T. S.; Linden, A.; Rogers, R. D. Linear Co-Ordinative Bonding at Oxygen: A Spectroscopic and Structural Study of Phosphine Oxide-Group 13 Lewis Acid Adducts. J. Chem. Soc., Dalton Trans. 1990, 1521-1528.

(53) Zaworotko, M. J.; Rogers, R. D.; Atwood, J. L. Interaction of Trimethylaluminum and Trimethylgallium with the Acetate Ion. Synthesis and Crystal Structures of $\left[\mathrm{Me}_{4} \mathrm{~N}\right]\left[\mathrm{Me}_{6} \mathrm{Al}_{2}(\mathrm{OAc})\right]$ and $\mathrm{Rb}\left[\mathrm{Me}_{6} \mathrm{Ga}_{2}(\mathrm{OAc})\right]$. Organometallics 1982, 1, 1179-1183.

(54) Cassidy, J. E.; Jarvis, J. A. J.; Rothon, R. N. Preparation, Characterisation, and Crystal and Molecular Structure of a Novel Tetrameric Aluminium Phosphate Complex: $\left[\left\{\mathrm{Al}\left(\mathrm{PO}_{4}\right)(\mathrm{HCl})\left(\mathrm{C}_{2} \mathrm{H}_{5} \mathrm{OH}\right)_{4}\right\}_{4}\right]$. J. Chem. Soc., Dalton Trans. 1975, 1497-1499.

(55) Robinson, G. H.; Lee, B.; Pennington, W. T.; Sangokoya, S. A. Facile Cleavage of C-H Bonds. Reaction of Trimethylaluminum with Bis(Diphenylphosphinoyl)Methane. Synthesis and Molecular Structure of $\left[\mathrm{Al}\left(\mathrm{CH}_{3}\right)\right]\left[\left(\mathrm{C}_{6} \mathrm{H}_{5}\right)_{2} \mathrm{P}(\mathrm{O}) \mathrm{CP}(\mathrm{O})\left(\mathrm{C}_{6} \mathrm{H}_{5}\right)_{2}\right]_{2}\left[\mathrm{Al}\left(\mathrm{CH}_{3}\right)_{2}\right]_{2}$. J. Am. Chem. Soc. 1988, $110,6260-6261$.

(56) Feher, F. J.; Budzichowski, T. A.; Weller, K. J. Polyhedral Aluminosilsesquioxanes: Soluble Organic Analogs of Aluminosilicates. J. Am. Chem. Soc. 1989, 111, 7288-7289.

(57) The absorption properties of complex 9a were not studied in the presence of 50 equivalents of phosphine oxide, as the absorbance properties of the ligand (Figure S16) would interfere with the spectrum of the complex.

(58) Frisch, M. J.; Trucks, G. W.; Schlegel, H. B.; Scuseria, G. E.; Robb, M. A.; Cheeseman, J. R.; Scalmani, G.; Barone, V.; Mennucci, B.; Petersson, G. A.; Nakatsuji, H.; Caricato, M.; Li, 
X.; Hratchian, H. P.; Izmaylov, A. F.; Bloino, J.; Zheng, G.; Sonnenberg, J. L.; Hada, M.; Ehara, M.; Toyota, K.; Fukuda, R.; Hasegawa, J.; Ishida, M.; Nakajima, T.; Honda, Y.; Kitao, O.; Nakai, H.; Vreven, T.; Montgomery, J., J. A. ; Peralta, J. E.; Ogliaro, F.; Bearpark, M.; Heyd, J. J.; Brothers, E.; Kudin, K. N.; Staroverov, V. N.; Kobayashi, R.; Normand, J.; Raghavachari, K.; Rendell, A.; Burant, J. C.; Iyengar, S. S.; Tomasi, J.; Cossi, M.; Rega, N.; Millam, J. M.; Klene, M.; Knox, J. E.; Cross, J. B.; Bakken, V.; Adamo, C.; Jaramillo, J.; Gomperts, R.; Stratmann, R. E.; Yazyev, O.; Austin, A. J.; Cammi, R.; Pomelli, C.; Ochterski, J. W.; Martin, R. L.; Morokuma, K.; Zakrzewski, V. G.; Voth, G. A.; Salvador, P.; Dannenberg, J. J.; Dapprich, S.; Daniels, A. D.; Farkas, Ö.; Foresman, J. B.; Ortiz, J. V.; Cioslowski, J.; Fox, D. J. 2013, Gaussian 09, Revision E.01, Gaussian Inc., Wallingford CT.

(59) Zhao, Y.; Truhlar, D. G. The M06 Suite of Density Functionals for Main Group Thermochemistry, Thermochemical Kinetics, Noncovalent Interactions, Excited States, and Transition Elements: Two New Functionals and Systematic Testing of Four M06-Class Functionals and 12 Other Functionals. Theor. Chem. Acc. 2008, 120, 215-241.

(60) Ernzerhof, M.; Scuseria, G. E. Assessment of the Perdew-Burke-Ernzerhof ExchangeCorrelation Functional. J. Chem. Phys. 1999, 110, 5029-5036.

(61) Vydrov, O. A.; Scuseria, G. E. Assessment of a Long-Range Corrected Hybrid Functional. J. Chem. Phys. 2006, 125, 234109-1-234109-9.

(62) Barbon, S. M.; Price, J. T.; Yogarajah, U.; Gilroy, J. B. Synthesis and Characterization of Conjugated/Cross-Conjugated Benzene-Bridged Boron Difluoride Formazanate Dimers. RSC $A d v .2015,5,56316-56324$.

(63) Chang, M.-C.; Chantzis, A.; Jacquemin, D.; Otten, E. Boron Difluorides with Formazanate Ligands: Redox-Switchable Fluorescent Dyes with Large Stokes Shifts. Dalton Trans. 2016, 45, 9477-9484. 
(64) The electrochemical properties of complex 9a were not studied in the presence of 50 equivalents of phosphine oxide due to the redox-activity of the ligand (Figure S20), which would overwhelm any current response arising from complex $9 \mathbf{a}$.

(65) Barbon, S. M.; Staroverov, V. N.; Gilroy, J. B. Effect of Extended $\pi$ Conjugation on the Spectroscopic and Electrochemical Properties of Boron Difluoride Formazanate Complexes. $J$. Org. Chem. 2015, 80, 5226-5235.

(66) Hesari, M.; Ding, Z. Review-Electrogenerated Chemiluminescence: Light Years Ahead. $J$. Electroelectrochem. Soc. 2016, 163, H3116-H3131.

(67) Nepomnyashchii, A. B.; Cho, S.; Rossky, P. J.; Bard, A. J. Dependence of Electrochemical and Electrogenerated Chemiluminescence Properties on the Structure of BODIPY Dyes. Unusually Large Separation between Sequential Electron Transfers. J. Am. Chem. Soc. 2010, $132,17550-17559$.

(68) Nepomnyashchii, A. B.; Bröring, M.; Ahrens, J.; Krüger, R.; Bard, A. J. Electrochemistry and Electrogenerated Chemiluminescence of $n$-Pentyl and Phenyl BODIPY Species: Formation of Aggregates from the Radical Ion Annihilation Reaction. J. Phys. Chem. C. 2010, 114, 1445314460.

(69) Maar, R. R.; Gilroy, J. B. Aggregation-Induced Emission Enhancement in Boron Difluoride Complexes of 3-Cyanoformazanates. J. Mater. Chem. C. 2016, 4, 6478-6482.

(70) Lee, S. B.; Kwon, J.; Kim, J. Enhanced Electrochemiluminescence of Luminol on Indium Tin Oxide Modified with Dendrimer-Encapsulated Au Nanoparticles. Electroanalysis 2015, 27, $2180-2186$. 


\section{For Table of Contents Only}

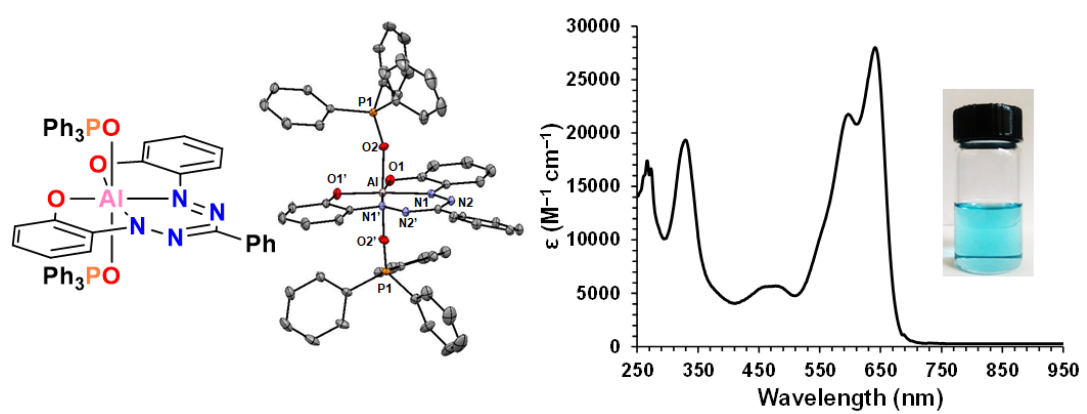

A new family of aluminum formazanate complexes were synthesized and characterized with multinuclear NMR spectroscopy, X-ray crystallography, cyclic voltammetry, and UV-vis absorption/emission spectroscopy. The electrochemiluminescence of one representative complex was probed in detail and DFT calculations were performed to rationalize the experimental findings. 